\title{
Uncertainty, Information Acquisition, and Price Swings in Asset Markets
}

\author{
ANTONIO MELE \\ Swiss Finance Institute, USI Lugano and CEPR \\ and \\ FRANCESCO SANGIORGI \\ Stockholm School of Economics
}

First version received March 2011; final version accepted March 2015 (Eds.)

\begin{abstract}
This article analyses costly information acquisition in asset markets with Knightian uncertainty about the asset fundamentals. In these markets, acquiring information not only reduces the expected variability of the fundamentals for a given distribution (i.e. risk). It also mitigates the uncertainty about the true distribution of the fundamentals. Agents who lack knowledge of this distribution cannot correctly interpret the information other investors impound into the price. We show that, due to uncertainty aversion, the incentives to reduce uncertainty by acquiring information increase as more investors acquire information. When uncertainty is high enough, information acquisition decisions become strategic complements and lead to multiple equilibria. Swift changes in information demand can drive large price swings even after small changes in Knightian uncertainty.
\end{abstract}

Key words: Asset Markets with Knightian Uncertainty, Asymmetric Information, Value of Parameter Uncertainty, Information Complementarities, Multiple Equilibria

JEL Codes: G12, G14, D82

\section{INTRODUCTION}

A basic tenet of financial economics holds that asset markets help summarize information dispersed across individual investors. But what information do asset prices transmit? Grossman and Stiglitz 1980) argue that as more agents acquire information, it becomes easier to free-ride on the (costly) learning of others merely by observing the asset price. The value of information diminishes with information acquisition.

The case for such a well-articulated role of the asset price relies on a number of assumptions that have become standard. This article relaxes what is arguably the most standard of them: that uncertainty can be quantified probabilistically. We consider a model in which the market fundamentals are subject to ambiguity, or Knightian uncertainty Keynes 1921; Knight 1921). In this market, aversion to ambiguity provides incentives to acquire information, incentives that 
increase with information acquisition. This property leads to a host of new conclusions about the informational role of asset prices.

Our results rely on a framework in which uninformed investors face Knightian uncertainty while attempting to glean information from the equilibrium price. We solve for the equilibrium in this asset market with asymmetric information, and then analyse endogenous information acquisition. The central element of our analysis is the agents' attitude vis-à-vis the information that prices reveal: how much of a price change can be attributed to new information, and how much to a liquidity shock? When uninformed investors are uncertain about the true distribution of the information held by the informed, this question cannot be given a precise probabilistic answer. In this market, the value of acquiring information has two components. The first accounts for the marginal value of reducing the riskiness of the fundamentals for any given prior distribution of returns - the standard Grossman and Stiglitz component. The second relates to how valuable it is for an ambiguity averse agent to resolve his ambiguity - the "value of parameter uncertainty". We show that the value of parameter uncertainty increases precisely as prices become more informative. If uncertainty is high enough, it dominates the standard Grossman-Stiglitz freeriding effect. Information complementarities result: the larger the mass of informed agents, the higher the benefits of becoming informed.

Why is resolving ambiguity more valuable when there are more informed agents? As it turns out, the value of parameter uncertainty lies in the benefit of forming portfolio decisions based on the true distribution of the information revealed by the price. This benefit is high precisely when prices incorporate more information on the fundamentals. Consider two polar cases:

In the first, no agent pays for information, and hence the equilibrium price at $t=1$, say, contains no information and is unambiguous (albeit risky because of liquidity trading) from an ex ante perspective (i.e. before trading and before the arrival of information, at $t=0$ ). However, returns from trading are ambiguous from an ex ante perspective because they amount to the ambiguous asset payoff less the unambiguous price. In this case, knowledge of the true return distribution at $t=1$ provides an informational advantage only when the true expected returns differ from those the uninformed investors impound into the equilibrium price. Ex ante, however, this informational advantage cannot be quantified probabilistically because the true return distribution is ambiguous. Thus, an ambiguity averse agent will give this advantage little weight as he formulates his choice over whether to pay for information. The value of parameter uncertainty is small.

At the other extreme, if all agents pay for information, then the price encodes important information about payoffs and is therefore ambiguous from an ex ante perspective. In contrast to the previous case, the anticipated returns are less ambiguous ex ante: they are the difference between the ambiguous payoff and the ambiguous price, and so the ambiguity "cancels out" as the price fully incorporates information about the asset payoff. However, knowledge about the probability distribution of the asset payoffs is valuable, because it provides the uninformed investors with a "code" for correctly interpreting the information conveyed through the price.

This feature of our model is illustrated by the following example. The three-color Ellsberg urn contains 30 red balls and a total of 60 black and yellow balls 1 Informed decision makers know the ratio of black to yellow balls, whereas the uninformed do not. At date-0, decision makers face a bet that pays 0 if a red ball is drawn and $\$ 1$ otherwise - a clearly unambiguous bet. The bet will be resolved at date-2. However, there is an interim date- 1 at which it will become known whether the drawn ball is black or not. If the drawn ball is black, then the date- 2 payoffs are known at date-1. However, if the drawn ball is not black, then at date-1 the decision maker knows that the drawn ball will be either red or yellow, but does not know which one it will be. Informed decision

1. Similar examples have been employed in the decision theory literature (e.g. Epstein and Schneider, 2003; Hanany and Klibanoff, 2007, 2009; Siniscalchi, 2011). 
makers can use Bayes' law and determine the probability of red or yellow, but the uninformed cannot, as the payoffs are no longer unambiguous. That is, learning about the ratio of black and yellow balls is valuable for forecasting the outcome of the bet precisely because of the information available at date-1.

In our model, the asset payoff is $f=\theta+\epsilon$, where $\theta$ is ambiguous (its mean is unknown), but can be learnt at some cost, and where $\epsilon$ has a known distribution. When all agents are informed, the price, $p$, moves one-to-one with the investors' private information $\theta$, that is $p=\theta-z$, where $z$ is the random asset supply. Asset returns, $R$, are unambiguous as a result, $R=f-p=\epsilon+z$. Even if the returns are unambiguous (tantamount to the unambiguous bet that places $\$ 1$ on "notred" in the urn example), $p$ reveals information that is useful for forecasting $R: R$ and $p$ have $z$ in common. However, $p$ contains ambiguous information, $\theta$, and is not very useful unless one knows the probability distribution of $\theta$ (just as the information "not-black" is not very useful unless one knows the original composition of balls in the urn in Ellsberg's example). For instance, a low realization of $p$ should not be interpreted as good news for future returns if the unknown mean of $\theta$ is very low (just as the information "not-black" does not imply high chances of getting \$1 if there are few yellow balls in the urn). An uninformed agent's portfolio decision will reflect his aversion to this uncertainty. However, whether the resulting decision correctly accounts for the true meaning of the price realization ultimately depends on the true distribution of $\theta$, which is ambiguous ex ante. Hence, while assessing the implications of remaining uninformed, an ambiguity averse investor fears making the wrong portfolio decision in light of price information. The value of parameter uncertainty is higher when prices incorporate information than when they do not.

A key prediction of our model is that in markets with ambiguity aversion, the value of parameter uncertainty increases when asset prices are sufficiently informative. Note that what is crucial is not merely the ambiguity, but the aversion to it. Critically, we show that in the smooth ambiguity extension of our baseline model (see below), higher-order uncertainty does not lead to information complementarities, unless agents are ambiguity averse.

It is well known that information complementarities can lead to multiple equilibria (Section5). Our model and its extensions do indeed predict multiple equilibria. Outcomes such as historydependent prices, market crashes, rebounds, and overshoots can result even from small changes in the uncertainty about the fundamentals. These properties help isolate new testable predictions regarding a largely unexplored issue: the market reaction to positive uncertainty shocks. Our model predicts that the initial reaction to a series of uncertainty shocks will be a market drop, led by reduced market participation, and followed by a sustained rally. The rally occurs because the increased uncertainty induces the uninformed agents to learn about these shocks, which fuels complementarities in information acquisition and price overshoots.

Our main model relies on a market in which agents have maxmin expected utility, as in Gilboa and Schmeidlen (1989). In this market, uninformed investors extract information from the equilibrium price through full Bayesian learning, by updating each initial prior. Uninformed investors are also sophisticated, in that they correctly anticipate their future choices (portfolio policies) in light of new information (the equilibrium price), an assumption that has been known as consistent planning since Strotz 1955-1956). However, our main conclusions are resilient to a variety of model extensions and alternative treatments of ambiguity, including (i) maximum likelihood updating, (ii) portfolio policies to which agents pre-commit before trading, and (iii) smooth specifications of ambiguity aversion as in Klibanoff, et al. 2005), which allow us to disentangle ambiguity from ambiguity aversion within the context of our study.

The article is organized as follows. The next section provides perspective on our contribution in light of the existing literature. Section 3 sets the model assumptions and analyses the asset market equilibrium. Section 4 characterizes the value of information in the asset market with 
Knightian uncertainty. Section 5 analyses endogenous information acquisition and deals with information complementarities, multiple equilibria, and the asset price swings that occur as a result. Section 6 provides the extensions. Section 7 concludes. The appendices contain details omitted from the main text. Proofs for Section 6 are in the Online Appendices D to F available as Supplementary Material.

\section{RELATED LITERATURE}

This article contributes to two strands of literature. First, it analyses how Knightian uncertainty about fundamentals affects assets and information markets in an otherwise standard noisy rational expectations equilibrium (REE) model. Secondly, it provides insights into the economic incentives for mitigating model uncertainty through costly information acquisition. To date, much of the literature on Knightian uncertainty does rely on a representative agent framework 2 on the other hand, the REE literature typically ignores the distinction between risk and ambiguity. Some exceptions are Caskey 2009) and Ozsoylev and Wernen 2011, who rely on noisy supply for partial revelation, as in our article, and Condie and Ganguli 2011,2012) and Easley et al. 2011), who do not. While these papers deal with informational properties of asset prices in markets with ambiguity, our focus is on the value of fundamental information in these markets.

In the existing REE literature, information complementarities can occur because as more agents acquire information, the price actually becomes less informative, making it more valuable for uninformed agents to acquire private information. This property can arise due to different mechanisms. In Barlevi and Veronesi 2008), it is a negative correlation between noisy supply and fundamentals. In Chamlev (2010), it is the possibility of independent jumps in noise trading and fundamentals. In Breon-Drish 2010), it is departures from normality of noise trading and fundamentals that lead to a failure of the monotone likelihood ratio property (MLRP) of the signal conveyed by the price 3 In Rahi and Zigrand 2014, the mechanism is the heterogeneity in agents' private asset valuations. In Avdis 2011 dynamic model, more informed investors lead to prices being more informative about dividends but less informative about the liquidity shocks that drive short-term price movements. Finally, Ganguli and Yang 2009) and Manzano and Vives 2011) show the existence of multiple linear equilibria in the price function when agents have private information about both dividends and supply. In one of these equilibria, the price signal-to-noise ratio decreases in the fraction of informed agents; when agents coordinate on this equilibrium, there are complementarities in information acquisition.

In contrast to the previous papers, information complementarities arise in our model despite the fact that more information acquisition leaves the uninformed agents with lower conditional risk and lower conditional ambiguity.

Other models also predict complementarities despite the price signal-to-noise ratio increasing with information acquisition. However, the mechanisms in these models hinge on different channels. In Veldkamp 2006, the mechanism relies on a cost of information that decreases with information demand. In García and Strob] 2011), the mechanism relies on consumption complementarities that result from relative wealth concerns. In the sequential trade model of Chamley 2007, , more informed trading makes current prices more informative but future prices more uncertain; the latter effect may increase the value of information for short-term investors.

2. Cao et al. 2005) and Easley and O'Hara 2009) contain early analyses of how ambiguity affects participation in Walrasian markets with heterogeneous agents.

3. Breon-Drish 2010) also provides a numerical example of complementarities in a setup in which the MLRP holds but uninformed agents' demands are backward-bending over some range. This feature of backward-bending demands further differentiates our article- the uninformed demand is downward sloping in all versions of our model. 
Our channel relies on the incentives to form portfolio decisions based on the true distribution of the fundamentals. Due to ambiguity aversion, these incentives increase when more investors acquire information and impound it into the asset price.

\section{THE MODEL}

\subsection{Agents and assets}

We consider a market for a risky asset, with payoff equal to $f=\theta+\epsilon$, where $\theta \sim N\left(\mu, \omega_{\theta}\right)$ and $\epsilon \sim$ $N\left(0, \omega_{\epsilon}\right)$. As in Grossman and Stiglit 1980), the market is populated by a continuum of agents, with a fraction $\lambda$ of informed and a fraction $1-\lambda$ of uninformed agents. Informed agents observe $\theta$ at cost $c>0$. We initially take $\lambda$ as given and consider endogenous information acquisition in the following sections. The asset supply is $z \sim N\left(\mu_{z}, \omega_{z}\right)$. We assume that $\omega_{\theta}>0$ (asymmetric information), $\omega_{\epsilon}>0$ (partial resolution of risk by private information), $\omega_{z}>0$ (partial information revelation), and that all variables are independent. A riskless asset is also available for trading; it is in perfectly elastic supply, and yields a rate of return equal to zero. All agents have negative exponential utility $u$ with constant absolute risk aversion $\tau$, that is $u(W)=-e^{-\tau W}$. Initial wealth is normalized to zero.

\subsection{Ambiguity and ambiguity aversion}

Our point of departure from Grossman and Stiglitz 1980) is the assumption that all agents are ex ante uncertain about the expected value of the fundamentals $f$. Although they are unable to assess what $\mu$ is, they believe it belongs to some interval, $\mu \in[\underline{\mu}, \bar{\mu}]$. We assume that $\underline{\mu}=$ $\mu_{0}-\frac{1}{2} \Delta \mu$ and $\bar{\mu}=\mu_{0}+\frac{1}{2} \Delta \mu$, for some $\Delta \mu \geq 0$, and set $\mu_{0}=0$.

In this and the following two sections, we assume that agents display preferences in the form of the maxmin expected utility model of Gilboa and Schmeidlen 1989). This assumption does not allow us to disentangle the notion of uncertainty from that of the attitude towards it. For example, we cannot tell whether an increased length of interval, $\Delta \mu$, reflects more uncertainty or more uncertainty aversion. Unless otherwise stated, we shall favour a cognitive interpretation of $\Delta \mu$. In Section 6.3 we rely on the smooth ambiguity model of Klibanoff, et al. 2005), which allows for a separation of tastes and beliefs. Our conclusions about complementarities and multiple equilibria remain unaffected in this framework 4

\subsection{Informed agents}

By observing the realization of $\theta$, informed agents resolve their ambiguity straight away. They choose portfolio holdings $x_{I}$ to maximize the expected utility of their final wealth $W_{I}=(f-p) x_{I}-$ $c$, where $p$ is the observed asset price. Standard arguments yield

$$
x_{I}(\theta, p)=\frac{E(f \mid \theta, p)-p}{\tau \operatorname{Var}(f \mid \theta, p)}=\frac{\theta-p}{\tau \omega_{\epsilon}} .
$$

Naturally, while informed agents are able to dissipate their ambiguity, they cannot eliminate risk. Conditional upon $\theta$, the fundamentals, $f$, are still normally distributed with expectation $\theta$ and variance $\omega_{\epsilon}$.

4. Other non-smooth models include, among others, the Choquet expected utility model of Schmeidlen 1989) and the $\alpha$-maxmin expected utility model of Ghirardato et al. 2004. The latter has the property of separating ambiguity and ambiguity attitude. Gilboa and Marinacci 2013) provide a survey of the decision-theoretic literature on ambiguity. 


\subsection{Uninformed agents}

Uncertainty about the expected value of the fundamentals, $\mu$, leads the uninformed agents to choose portfolio holdings so as to maximize

$$
\min _{\mu \in[\underline{\mu}, \bar{\mu}]} E_{\mu}\left(-e^{-\tau W_{U}} \mid p\right)=-e^{-\tau \min _{\mu \in[\underline{\mu}, \bar{\mu}]} E_{\mu}\left(W_{U} \mid p\right)+\frac{1}{2} \tau^{2} \operatorname{var}\left(W_{U} \mid p\right)},
$$

where $W_{U}=(f-p) x_{U}, x_{U}$ is the asset demand, and $E_{\mu}(\cdot)$ is the expectation operator taken under the assumption that $E(\theta)=\mu$.

The criterion underlying Equation (3.2) is the maxmin expected utility axiomatized by Gilboa and Schmeidlen 1989). While formulating portfolio decisions, agents learn from the price and update each of their beliefs. This rule is known as full Bayesian updating. It was proposed by Jaffray 1992), among others, and axiomatized by Pires 2002). In Section 6.1 we solve for an alternative updating rule, based on the maximum likelihood principle. This alternative assumption does not affect our main conclusions on information acquisition based on full Bayesian updating.

We conjecture that, for every pair $(\theta, z)$, the equilibrium price function is $P(\theta, z)$. We look for an equilibrium in which the uninformed agents sell the asset when the price is sufficiently high and buy the asset when the price is sufficiently low, in a sense made precise below. This search process leads to a simpler problem, that of determining the expectation of the fundamentals when the agents buy and when the agents sell. Accordingly, we introduce the following notation:

$$
E^{\text {buy }}(f \mid P(\cdot, \cdot)=p) \equiv E_{\underline{\mu}}(f \mid P(\cdot, \cdot)=p), \quad E^{\text {sell }}(f \mid P(\cdot, \cdot)=p) \equiv E_{\bar{\mu}}(f \mid P(\cdot, \cdot)=p)
$$

We conjecture that the solution to the uninformed agents' problem is

$$
x_{U}(p, P(\cdot, \cdot))= \begin{cases}\frac{E^{\mathrm{buy}}(f \mid P(\cdot, \cdot)=p)-p}{\tau \operatorname{Var}(f \mid P(\cdot, \cdot)=p)}, & \text { for } p<E^{\mathrm{buy}}(f \mid P(\cdot, \cdot)=p) \\ 0, \quad \text { for } p \in\left[E^{\mathrm{buy}}(f \mid P(\cdot, \cdot)=p), E^{\operatorname{sell}}(f \mid P(\cdot, \cdot)=p)\right] \\ \frac{E^{\mathrm{sell}}(f \mid P(\cdot, \cdot)=p)-p}{\tau \operatorname{Var}(f \mid P(\cdot, \cdot)=p)}, & \text { for } p>E^{\operatorname{sell}}(f \mid P(\cdot, \cdot)=p)\end{cases}
$$

That is, the uninformed agents do not participate in the market unless the equilibrium price is "favorable" enough. Precisely, the uninformed agents enter the market as buyers (sellers) only if the price realization, $p$, is lower (higher) than the agents' worst-case scenario expectation of the asset value, conditional on $p$. Hence, participation involves a fixed-point problem in which the expectation of the asset value, conditional on the price realization, is equal to the same price realization:

$$
E^{\text {buy }}(f \mid P(\cdot, \cdot)=\underline{p})=\underline{p} \text { and } E^{\text {sell }}(f \mid P(\cdot, \cdot)=\bar{p})=\bar{p}
$$

The uninformed agents buy the asset when the price realization, $p$, is below $p$, and sell the asset when $p$ is above $\bar{p}$. They do not trade if the price realization is such that $p \in[p, \bar{p}]$. Such situations of portfolio inertia were first linked with Knightian uncertainty by Dow and Werlang (1992). In our model, the cut-offs $p$ and $\bar{p}$ are determined in equilibrium, and so is the extent of the uninformed agents' market participation. 


\subsection{Equilibrium}

We conjecture that the equilibrium price function is $P(\theta, z)=P(s(\theta, z))$, where $s(\theta, z)$ is the compound signal, defined as

$$
s(\theta, z)=\frac{\lambda}{\tau \omega_{\epsilon}} \theta-\left(z-\mu_{z}\right) .
$$

From the market-clearing condition,

$$
(1-\lambda) x_{U}(p, P(\cdot))+\lambda x_{I}(\theta, p)=z,
$$

we see that the compound signal is observationally equivalent to the equilibrium price. Therefore, the equilibrium in this market is also one in which uninformed agents condition the expectation of the asset value upon the observation of the compound signal.

We have:

Proposition 1. (Asset market equilibrium). The equilibrium price is piecewise linear in the compound signal s given in equation (3.5), as follows:

$$
P(s)= \begin{cases}\underline{a}+b s, & \text { for } s<\underline{s} \\ a+\frac{\tau \omega_{\epsilon}}{\lambda} s, & \text { for } s \in[\underline{s}, \bar{s}] \\ \bar{a}+b s, & \text { for } s>\bar{s}\end{cases}
$$

for constants $\underline{a}, \bar{a}, a, b$ given in Appendix A. The threshold values for the compound signal, $\underline{s}, \bar{s}$, satisfy

$$
\underline{s}=\frac{\lambda}{\tau \omega_{\epsilon}} \underline{\mu}+\frac{\omega_{s}}{\omega_{z}} \mu_{z}, \quad \bar{s}-\underline{s}=\frac{\lambda}{\tau \omega_{\epsilon}} \Delta \mu,
$$

where $\omega_{s}$ is the variance of s. Finally, we have $\underline{p}=\underline{a}+b \underline{s}$ and $\bar{p}=\bar{a}+b \bar{s}$, with $\underline{p}<\bar{p}$.

Figure 1 depicts the equilibrium price described in Proposition 1 when the fraction of informed agents is, respectively, $\lambda=0.2$ (top panel) and $\lambda=0.5$ (bottom). The solid line is the price in the presence of ambiguity, $\Delta \mu>0$, and the dashed line is the price in the Grossman and Stiglitz 1980) model.

The portfolio choice given in equation (3.3) reflects returns expected in worst-case scenarios. In equilibrium, the uninformed agents buy if $s<\underline{s}$ ( $s e l l$ if $s>\bar{s}$ ) but they buy or sell less than they would do in the absence of ambiguity. As a result, the price is lower (higher) than in Grossman and Stiglitz for low (high) realizations of the compound signal, $s$. Naturally, the price impact of the uninformed agents is reduced as $\lambda$ increases.

When the signal realization lies within the range $[\underline{s}, \bar{s}]$, the uninformed agents do not participate. Note that both $\underline{s}$ and $\bar{s}$ increase with the average asset supply $\mu_{z}$, reflecting an increased probability that the uninformed agents will be buyers. Proposition 1 also tells us that the threshold difference, $\bar{s}-\underline{s}$, equals the size of the ambiguity, $\Delta \mu$, weighted by the risk-bearing capacity of the informed

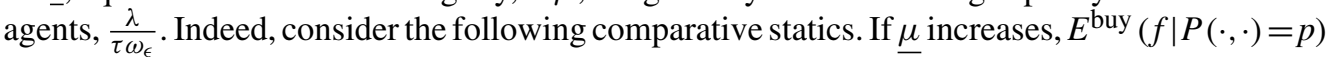
increases as well, for each price realization $p$. Thus, the threshold equilibrium price $p$ has to increase (and, hence, so does $\underline{s}$ ), according to the fixed-point problem in equation (3.4); similarly, $\bar{s}$ decreases as $\bar{\mu}$ decreases.

While the size of the non-participation region is proportional to the informed risk-bearing capacity, the comparative statics of the probability of non-participation for a fixed prior $\mu \in[\underline{\mu}, \bar{\mu}]$ 
Equilibrium price, $\lambda=0.2$

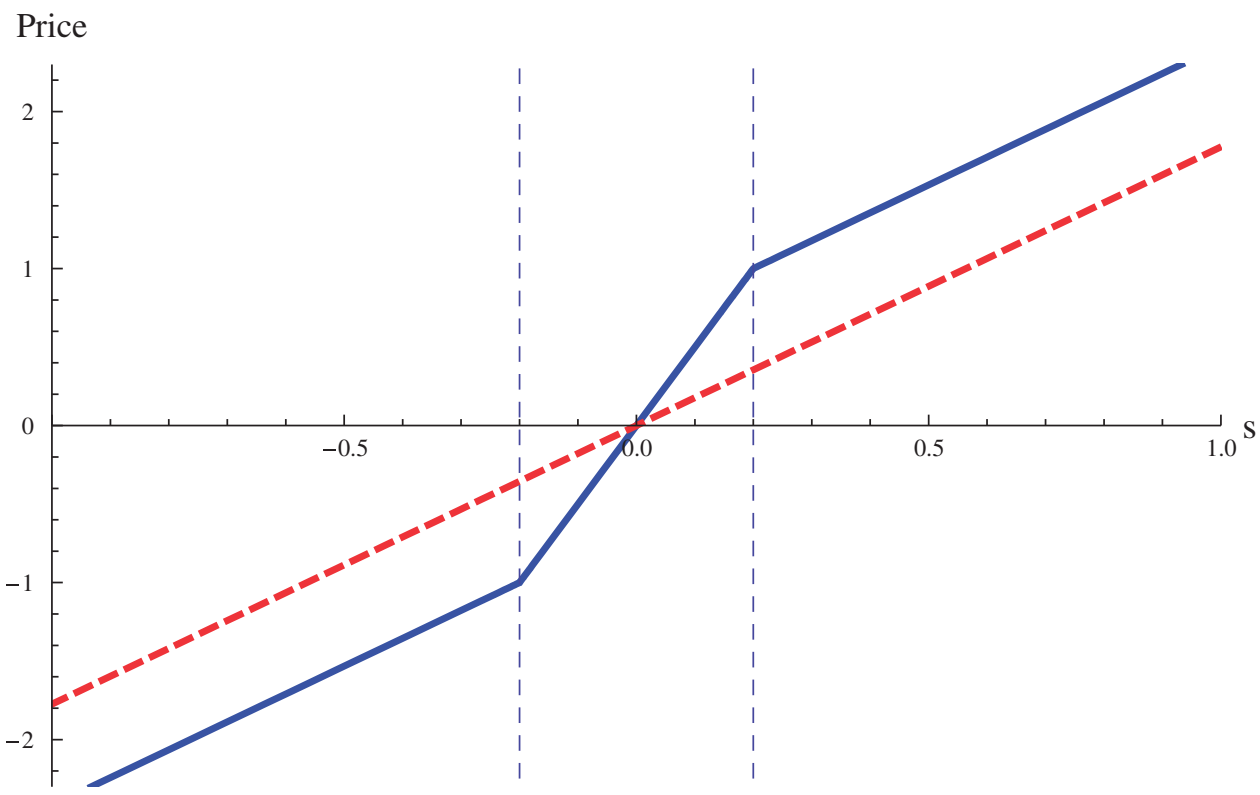

Equilibrium price, $\lambda=0.5$

Price

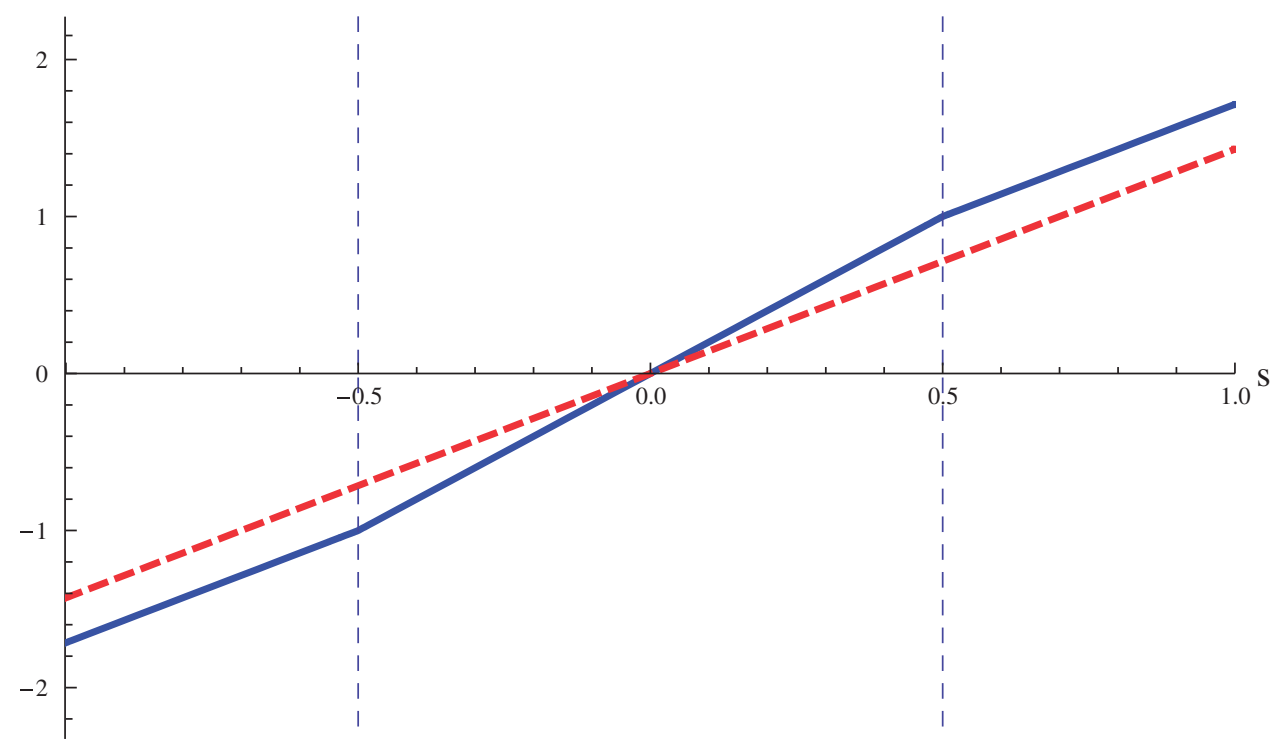

FIGURE 1

This figure depicts the equilibrium asset price given in Proposition 1 as a function of the compound signal, $s$. Both panels compare the price function with the Grossman-Stiglitz linear function (the dashed line), which arises in the absence of ambiguity, that is, when $\Delta \mu=0$. The region delimited by the vertical dashed lines corresponds to the uninformed agents' non-participation region. The parameter values are $\Delta \mu=2, \omega_{\theta}=\omega_{\epsilon}=\omega_{z}=\tau=1$, and $\mu_{z}=0$. In the top panel, the proportion of informed agents, $\lambda=0.2$, and in the bottom panel, $\lambda=0.5$. 
can display non-monotonicities depending on parameter values. This probability is determined by both the size of the non-participation region, $\bar{s}-\underline{s}$, and the distribution of the signal defined in equation 3.5, both of which depend on the informed risk-bearing capacity, $\frac{\lambda}{\tau \omega_{\epsilon}}$. For example, larger values of $\lambda$ increase both the non-participation region and the variance of the signal. However, if the average asset supply $\mu_{z}$ is small, then a more volatile signal (for a fixed $\bar{s}-\underline{s})$ implies that non-participation is less likely. When $\lambda$ is small, the signal's volatility has second-order effects, such that the probability of non-participation increases with $\lambda$. When $\lambda$ is sufficiently high, the effects of the signal's variability may dominate, such that the probability of non-participation could decrease with $\lambda$.

\section{THE VALUE OF INFORMATION}

This section analyses how ambiguity affects the incentives to acquire fundamental information. Agents decide whether to become informed before trading, as in Grossman and Stiglitz (1980), and assess their information choices based at worst-case scenarios. The value of acquiring information reflects not only a reduction in the payoff risk, but also the agents' aversion to ambiguity. We decompose the value of information into two components that summarize these two effects.

\subsection{Informed agents}

Acquiring private information allows an agent to observe the ambiguous portion of the asset payoff. Thus, information acquisition reduces the conditional risk and eliminates the conditional ambiguity of the payoff. Because information is only known after it has been paid for, a would-be informed agent faces ambiguity ex ante regarding the distribution of the $\theta$ that he will observe at the trading stage. In Appendix B, we show that his ex ante utility is,

$$
\mathcal{U}_{I}(c, \lambda)=\min _{\mu \in[\underline{\mu}, \bar{\mu}]} E_{\mu}\left[-e^{-\tau \hat{W}_{I}}\right]=e^{\tau c} \sqrt{\frac{\omega_{f \mid \theta}}{\omega_{f \mid s}}} \cdot \overline{\mathcal{U}}_{U}(\lambda),
$$

where $\hat{W}_{I}$ is the wealth generated by the portfolio choice given by equation $\underline{3.1}, \omega_{f \mid \theta}=\omega_{\epsilon}$, and $\omega_{f \mid s}$ is the variance of $f$ conditional on $s$ (equation (5.1)); finally, the two expressions,

$$
\overline{\mathcal{U}}_{U}(\lambda) \equiv \min _{\mu \in[\underline{\mu}, \bar{\mu}]} E_{\mu}\left[-e^{-\tau \overline{\mathcal{C}}(s ; \mu)}\right], \quad-e^{-\tau \overline{\mathcal{C}}(s ; \mu)}=\max _{x} E_{\mu}\left[-e^{-\tau x(f-p)} \mid s\right],
$$

denote the ex ante utility $\left(\overline{\mathcal{U}}_{U}\right)$ and the expected utility at the trading stage $\left(-e^{-\tau \overline{\mathcal{C}}}\right)$ conditional only on (i) the equilibrium price $p$ (equivalently, the compound signal $s$ ) and (ii) full knowledge (at the trading stage) of the otherwise ambiguous expected value of $\theta, \mu$. Equivalently, $\overline{\mathcal{C}}$ is the certainty equivalent at the trading stage for a hypothetical uninformed agent who only faces risky choices. This representation of the informed utility plays a crucial role in explaining the main determinants of information acquisition. Appendix B provides closed-form expressions for the expectations in equation 4.2 and the certainty equivalent $\overline{\mathcal{C}}$.

\subsection{Uninformed agents and consistent planning}

An agent who remains uninformed anticipates making portfolio choices while lacking knowledge of the payoff distribution. Aversion to this uncertainty affects his ex ante utility. We describe this 
effect in terms of a welfare loss with respect to the benchmark in which the agent only faces risky portfolio choices, $\overline{\mathcal{U}}_{U}(\lambda)$ in equation 4.2 .

Let us denote by $x_{U}(s) \equiv x_{U}(p, P(\theta, z))$ the portfolio choice of the uninformed agent in equation (3.3), and by $\bar{x}_{U}(s ; \mu)$ the solution to the maximization problem in equation [4.2),

$$
x_{U}(s)=\bar{x}_{U}(s ; \mu)+\frac{E_{\mu^{*}(s)}(f \mid s)-E_{\mu}(f \mid s)}{\tau \omega_{f \mid s}}, \quad \mu^{*}(s)=\arg \min _{\mu \in[\underline{\mu}, \bar{\mu}]}\left|E_{\mu}(f \mid s)-P(s)\right| .
$$

Note that $E_{\mu^{*}(s)}(f \mid s)$ is the worst-case scenario estimate of the asset value that the uninformed agent formulates while trading in state $s\left(\right.$ e.g. $\mu^{*}(s)=\mu$ when the agent buys; Section 3). While evaluating his ex ante welfare, the uninformed agent anticipates that his future portfolio choice in state $s$ will reflect such a worst-case scenario estimate. When gauged through a generic prior $\mu$, this estimate is deemed biased, in that it leads to a portfolio decision that conflicts with the choice he would have made had he known $\mu$ at the trading stage. Clearly, $x_{U}(s) \neq \bar{x}_{U}(s ; \mu)$ in all states $s$ in which $\mu^{*}(s)$ differs from $\mu$. In Appendix B, we show that this bias can be accounted for as a discount on $\overline{\mathcal{C}}(s ; \mu)$. Precisely, we show that the ex ante utility of an uninformed agent can be expressed as

$$
\mathcal{U}_{U}(\lambda)=\min _{\mu \in[\underline{\mu}, \bar{\mu}]} E_{\mu}\left[-e^{-\tau \hat{W}_{U}}\right]=\min _{\mu \in[\underline{\mu}, \bar{\mu}]} E_{\mu}\left[-e^{-\tau(\overline{\mathcal{C}}(s ; \mu)-\mathcal{T}(s ; \mu))}\right],
$$

where $\hat{W}_{U}$ denotes the wealth generated by the portfolio choice given in equation 3.3 , and

$$
\mathcal{T}(s ; \mu) \equiv \frac{1}{2 \tau \omega_{f \mid s}}\left(E_{\mu^{*}(s)}(f \mid s)-E_{\mu}(f \mid s)\right)^{2} .
$$

The term inside the expectation in the second equality of equation 4.4 is the expectation under prior $\mu$ of terminal utility conditional on price information. It has two terms: one accounting for the risk-only profit certainty equivalent $\overline{\mathcal{C}}(s ; \mu)$ in equation 4.2 , and another relating to ambiguity effects, $\mathcal{T}(s ; \mu)$. If the agent knew the true distribution at the trading stage (i.e. $\mu)$, $x_{U}(s)$ in equation (4.3) would collapse to $\bar{x}_{U}(s ; \mu)$ or, equivalently, the discount $\mathcal{T}(s ; \mu)$ would be trivially zero, and $\mathcal{U}_{U}(\lambda)$ would collapse to $\overline{\mathcal{U}}_{U}(\lambda)$. The term $\mathcal{T}(s ; \mu)$ is given a closed-form expression in Appendix B (equation (B.11). It links to the intertemporal tussles described by Strot 1955-1956), in contexts where preferences may conflict at different decision points.

Violation of dynamic consistency is a well-known phenomenon arising in contexts with ambiguity Epstein and Schneiden 2003). Appendix B illustrates that in our model with asymmetric information, there exist alternative state-contingent portfolio policies to $x_{U}(\cdot)$ in equation (4.3), yielding an ex ante utility higher than $\mathcal{U}_{U}(\lambda)$. Intuitively, a state-contingent portfolio policy $x_{U}(s)$ could be chosen such that the uninformed agents could buy or sell more than indicated by equation 4.3 and achieve a higher ex ante utility (see the parametric example in Appendix B, equation (B.13). The loss $\mathcal{T}(s ; \mu)$ arises because the uninformed agents take into account that their state-contingent portfolio policy deviates from the one they would formulate ex ante. In other words, agents are sophisticated, in that they adopt a strategy described by Strotz 1955-1956) as one of consistent planning 5 Committing to a predetermined portfolio policy is an alternative way of dealing with these intertemporal conflicts. This strategy is described by Strotz as one of pre-commitment. In Section 6.2 we extend our results to a model with pre-commitment.

5. Siniscalchi 2011) develops a decision-theoretic treatment of consistent planning based on trees rather than temporal acts. His approach goes beyond the "multiple-selves" approach introduced by Strotz to deal with intertemporal conflicts. 


\subsection{Equilibrium demand for information}

The value of acquiring private information can be decomposed into two parts: one relating to the benefits of resolving ambiguity before trading, and another summarizing the value of learning the realization of $\theta$ in addition to the unknown distribution (i.e. $\mu$ ) before trading. Let $\mathcal{G}(c, \lambda) \equiv$ $-\frac{1}{\tau} \ln \left(\frac{\mathcal{U}_{I}(c, \lambda)}{\mathcal{U}_{U}(\lambda)}\right)$ denote the net gain from becoming informed, defined as the difference between the ex ante profit certainty equivalent of the informed and that of the uninformed. By equations 4.1), 4.2, and 4.4, it is given by

$$
\begin{aligned}
\mathcal{G}(c, \lambda) & =-\frac{1}{\tau} \ln \left(\frac{\mathcal{U}_{I}(c, \lambda)}{\overline{\mathcal{U}}_{U}(\lambda)}\right)-\frac{1}{\tau} \ln \left(\frac{\overline{\mathcal{U}}_{U}(\lambda)}{\mathcal{U}_{U}(\lambda)}\right) \\
& =\underbrace{\frac{1}{2 \tau} \ln \left(\frac{\omega_{f \mid s}}{\omega_{f \mid \theta}}\right)-c}_{\text {Grossman-Stiglitz effect }}+\underbrace{\frac{1}{\tau} \ln \left(\frac{E_{\mu_{U}}\left[e^{-\tau\left(\overline{\mathcal{C}}\left(s ; \mu_{U}\right)-\mathcal{T}\left(s ; \mu_{U}\right)\right)}\right]}{E_{\mu_{I}}\left[e^{-\tau \overline{\mathcal{C}}\left(s ; \mu_{I}\right)}\right]}\right)}_{\text {Value of parameter uncertainty }},
\end{aligned}
$$

where $\mu_{I}$ and $\mu_{U}$ solve the two problems described in equations (4.1) and 4.4.

The R.H.S. of equation 4.6 measures the value of information, net of the cost $c$, for a given fraction $\lambda$ of informed agents. An equilibrium with endogenous information acquisition is defined in the standard way. An interior equilibrium is a fraction of informed agents $\lambda^{*} \in(0,1)$ that causes any agent to be ex ante indifferent over whether to be informed or not, that is, $\mathcal{G}\left(c, \lambda^{*}\right)=0$. The non-interior equilibria are $\lambda^{*}=0$ such that $\mathcal{G}(c, 0) \leq 0$, and $\lambda^{*}=1$ such that $\mathcal{G}(c, 1) \geq 0$.

The value of information has two components, as anticipated. The first is the marginal value of reducing the riskiness of the fundamentals for any given prior distribution of returns. It is monotonically decreasing in $\lambda$, as explained below (equation 5.1). It actually coincides with the standard Grossman and Stiglitz 1980) value of information, summarizing the usual trade-off between the cost of acquiring information and its benefits in a market without ambiguity, that is, where $\Delta \mu=0$.

The second component, labelled "value of parameter uncertainty," summarizes how valuable it is for an ambiguity averse agent to resolve ambiguity before trading. It measures the ex ante value to an uninformed agent of making future portfolio choices based on the true probability distribution (as opposed to worst-case scenarios) when confronted with price information. This value links to the uninformed ex ante evaluation of the welfare loss $\mathcal{T}(s ; \mu)$ implied by his portfolio choice. Note that if $\mathcal{T}\left(s ; \mu_{U}\right)$ were zero in all states, then the value of parameter uncertainty would be nil because the informed agents' welfare is also appraised based on the worst-case scenario. However, the next proposition establishes that this value is always positive:

Proposition 2. (Value of parameter uncertainty). Let $\Delta \mu>0$. Then, the value of parameter uncertainty in equation (4.6) is strictly positive. That is, information is more valuable in a market with ambiguous fundamentals $(\Delta \mu>0)$ than in a market without ambiguity $(\Delta \mu=0)$.

One implication of Proposition 2 is that the amount of resources spent on collecting information is higher in markets with ambiguity than in markets without, as formalized by Corollary B.1 in Appendix B.

Which component of the value of information in equation (4.6) becomes most relevant as more agents acquire information? It is an important issue, as the value of parameter uncertainty can dwarf the familiar Grossman-Stiglitz effect and result in information complementarities: the 
higher the number of informed agents, the higher the incentives to acquire information. These issues are analysed next.

\section{INFORMATION ACQUISITION}

\subsection{Information revelation}

What information does the asset price transmit to uninformed agents? How much information does the price reveal as the number of informed agents increases? Regarding risk, Appendix B (equation (B.6) shows that the variance of the fundamental, conditional on price information, is as in Grossman and Stiglitz 1980, namely

$$
\operatorname{var}(f \mid P(\cdot)=p) \equiv \omega_{f \mid s}=\omega_{\varepsilon}+(1-\chi) \omega_{\theta}, \quad \chi=\frac{\left(\frac{\lambda}{\omega_{\epsilon} \tau}\right)^{2} \omega_{\theta}}{\left(\frac{\lambda}{\omega_{\epsilon} \tau}\right)^{2} \omega_{\theta}+\omega_{z}} .
$$

Regarding ambiguity conditional on price information,

$$
E_{\bar{\mu}}(f \mid P(\cdot)=p)-E_{\underline{\mu}}(f \mid P(\cdot)=p)=(1-\chi) \Delta \mu \leq \Delta \mu \equiv E_{\bar{\mu}}(f)-E_{\underline{\mu}}(f),
$$

where the first equality follows from equations A.2 A.3 in Appendix A. The L.H.S. of the equality in equation (5.2) is the uncertainty remaining after an agent has observed the equilibrium price. It is less than the unconditional ambiguity, $\Delta \mu$. That is, price information helps reduce ambiguity. Due to noisy supply $\left(\omega_{z}>0\right)$, information revelation is partial. Furthermore, since the parameter $\chi$ in equation (5.1) is increasing in $\lambda$, the model predicts that information acquisition always leads to more informative prices, in terms of both risk and ambiguity. Despite the latter intuitive property, the next section shows that due to ambiguity aversion, information complementarities arise in this market.

\subsection{Ambiguity aversion and information complementarities}

The value of private information can be split into the benefit of reducing risk and the value of parameter uncertainty (equation (4.6). The latter is the value an uninformed agent would be willing to pay ex ante for making future trading decisions based on the true distribution of the fundamentals. This value increases as the price becomes more informative. In the following examples, we provide intuition by comparing the welfare of an uninformed agent, $\mathcal{U}_{U}(\lambda)$, to that of a hypothetical uninformed agent who only faces risky choices at the trading stage, $\overline{\mathcal{U}}_{U}(\lambda)$, as defined in Section 4.1

Consider two polar cases in which either a few agents are informed or many are. To simplify exposition, we assume that the asset is in positive supply. Define the equilibrium asset return per share as the difference between the fundamentals $f$ and the price $P(s)$ given by equation 3.7),

$$
R \equiv \theta+\epsilon-P(s(\theta, z)) .
$$

When there are no informed agents, the price does not incorporate information on the ambiguous $\theta$. Because an uninformed agent's portfolio choice ( $x_{U}$ in equation (3.3) is only a function of the price, there is no ambiguity regarding the choice he will make. That is, he will face ambiguity at the trading stage, and evaluate the asset based on the worst-case scenario $\mu^{*}(s)=\underline{\mu}$; however, his actual portfolio choice will only depend on the realization of the asset 
supply (not $\theta$ ). Profits from trading, $x_{U} \cdot R$, are ex ante ambiguous though. Indeed, the price is uninformative and the expected returns are unknown, depending as they do on the unknown mean of the fundamentals, $E_{\mu}(R)=\mu-E(P)$. Anticipating holding the per-capita supply, the ambiguity averse uninformed agent will then appraise the expected returns under the worst-case scenario $\mu_{U}=\mu$.

What is the ex ante value of resolving ambiguity regarding $\mu$ in this case? On the one hand, the agent would make portfolio decisions based on the true (not worst-case) expected return. He would then benefit from an informational advantage that would grow with $\mu-\mu \geq 0$, the difference between the true $\mu$ and the prior impounded into the price by the uninformed agents, $\underline{\mu}$. On the other hand, the true $\mu$ is unknown when information decisions are made. Thus, the agent's ambiguity aversion makes him evaluate (ex ante) this advantage based on the worstcase scenario $\mu_{I}=\mu$. That is, the informed agent anticipates making exactly the same portfolio choice and profits as the uninformed agent. Resolving ambiguity regarding $\mu$ has no value in this example. Formally, we have:

Lemma 1. In a market with no informed agents, i.e. where $\lambda=0$, there exists a $\bar{\mu}_{z}>0$ such that for all $\mu_{z} \geq \bar{\mu}_{z}$, the ex ante prior of both the uninformed and the informed agents is $\mu_{U}=\mu_{I}=\mu$.

In other words, when prices are not very informative, and the average supply $\mu_{z}$ is high enough 6 the ex ante beliefs of the informed and the uninformed are the same. The beliefs coincide with the uninformed worst-case scenario at the trading stage, $\mu_{I}=\mu_{U}=\mu^{*}(s)=\underline{\mu}$, such that the welfare loss $\mathcal{T}\left(s ; \mu_{U}\right)=0$ in the most likely region of the state space. The value of parameter uncertainty in equation 4.6 is small as a result.

As the number of informed agents increases, the price becomes more responsive to changes in $\theta$. Consider the limiting case in which everyone is informed, as in the example provided in the Introduction. In this case, the price fully incorporates information on the fundamentals, such that returns become unambiguous albeit still risky: $P=\theta-\tau \omega_{\epsilon} z$, and $R=\epsilon+\tau \omega_{\epsilon} z$. Returns are independent of $\theta$ (and thus unambiguous). Yet, lacking knowledge of the distribution of $\theta$, the uninformed agent would now be exposed to ambiguous profits, $x_{U} \cdot R$, through his own portfolio choices, $x_{U}$. How this happens is easily seen. Note that $P$ still contains information about $R$. Thus, while formulating his portfolio decision $x_{U}$ in equation 3.3 , the uninformed agent forms his prior-to-prior estimate of $R$ given the price:

$$
E_{\mu}(R \mid p)=E(R)+\frac{\operatorname{cov}(R, p)}{\operatorname{var}(p)}\left(p-E_{\mu}(p)\right)
$$

Equation (5.4) allows us to elaborate on the question raised in the Introduction: "How much of a price change can be attributed to new information, and how much to a liquidity shock?" To give a precise probabilistic answer, the true mean of $p$ must be known. Lack of this knowledge leads an uninformed agent to update his beliefs based on worst-case scenarios: the agent's expectation $E_{\mu}(p)$ in equation 5.4 is $E_{\mu^{*}(s)}(p)$, where $\mu^{*}(s)=\mu$ when the agent is long the asset and $\mu^{*}(s)=\bar{\mu}$ when he is short. This makes the distribution of $p-E_{\mu^{*}(s)}(p)$ (and therefore the

6. When $\mu_{z}$ is high, there is a high probability that an uninformed agent will be long the asset, which matches the intuition in these examples with positive asset supply. By contrast, because the asset supply is Gaussian in the model, lower values of $\mu_{z}$ increase the probability of the event that the uninformed agents are short the asset. In this event, the prior incorporated into the price is $\bar{\mu}$, such that the informed agent anticipates making a different portfolio choice than the uninformed agent. The ensuing informational advantage is small (it is assessed at worst-case scenario) but positive. A large $\mu_{z}$ facilitates our proofs, although it is not needed to generate complementarities, as we explain below (Figure 2 . 
distribution of his own portfolio choice) dependent on $\mu$, which is unknown at the time of making the information decision.

How does the agent gauge the welfare implications of remaining uninformed? The agent's ambiguity aversion leads him to evaluate his future portfolio choice according to the worstcase scenario, that is, as if his beliefs at the trading stage will be "unwarranted". Precisely, the uninformed ex ante beliefs are $\mu_{U}=\bar{\mu}$ if the probability of being long (and, hence, that $\mu^{*}(s)=\mu$ at the trading stage) is sufficiently high; by contrast, the agent's utility is independent of $\mu$ if he becomes informed. The ensuing welfare loss, $\mathcal{T}\left(s ; \mu_{U}\right)>0$, is what makes the value of parameter uncertainty positive in equation 4.6. The following lemma summarizes our conclusions regarding the agents' ex ante beliefs in these informationally rich markets:

Lemma 2. There exists a $\bar{\mu}_{z}>0$ and a level of information acquisition $\bar{\lambda} \in(0,1)$ depending on $\mu_{z}$, such that for all $\mu_{z} \geq \bar{\mu}_{z}$, the ex ante prior of the uninformed agents is $\mu_{U}=\bar{\mu}$ for all $\lambda \in(\bar{\lambda}, 1]$. Moreover, for $\lambda=1$, the ex ante utility of the informed agents is independent of $\mu$.

Lemmas 1 and 2 say that the prior of the uninformed agents' makes a switch from low $\left(\mu_{U}=\mu\right)$ to high $\left(\mu_{U}=\bar{\mu}\right)$ as markets become more informative. Thus, they suggest that the value of parameter uncertainty increases from zero to positive as the number of informed agents increases. Naturally, the fact that the value of parameter uncertainty increases with information acquisition does not imply information complementarities. Complementarities arise once uncertainty is high enough for this increase to dominate the Grossman-Stiglitz effect in equation 4.6):

Proposition 3. (Information complementarities). There exist a level of uncertainty $\overline{\Delta \mu}>0$ and an average asset supply $\bar{\mu}_{z}>0$, such that there are complementarities in information acquisition for all $\Delta \mu>\overline{\Delta \mu}$ and $\mu_{z}>\bar{\mu}_{z}$.

The left panel of Figure 2 illustrates Proposition 3 For the value of information to be higher in $\lambda=1$ than in $\lambda=0, \Delta \mu$ needs to be large enough. However, progressively lower values of $\Delta \mu$ are needed as $\mu_{z}$ increases. Indeed, a higher probability of being a buyer lowers the value of parameter uncertainty when $\lambda=0$ and increases it when $\lambda=1$ as discussed previously 7 Therefore, when $\mu_{z}$ is large, the increase in the value of parameter uncertainty dwarfs the Grossman-Stiglitz effect even when $\Delta \mu$ is small.

The right panel of Figure 2 depicts the loci of $\Delta \mu$ and $\mu_{z}$ such that information complementarities arise for any open interval of $\lambda$. The pattern and its rationale are the same as in the left panel with two additional features. First, when $\mu_{z}$ is sufficiently large, information complementarities are triggered by lower values of $\lambda$ as $\Delta \mu$ increases, as will be further explained in Section 5.4 (Figure 3). Secondly, complementarities arise also when the asset is in zero average supply, $\mu_{z}=0$, but for low values of $\lambda$.

The intuition behind the first feature is the same as that underlying the left panel of Figure 2. The value of parameter uncertainty is small when $\lambda=0$, but it increases even after a small increase in $\lambda$, provided $\Delta \mu$ is high enough.

The intuition behind the second feature is as follows. When the average asset supply is small or zero and $\lambda$ is small, an uninformed agent has comparable odds of being either a buyer or a seller. When the number of informed agents grows, an uninformed agent now faces a higher chance of not participating at all, as explained in Section 3 The value of parameter uncertainty increases as a result of foregone investment opportunities. Moreover, opportunities that are foregone due to not 

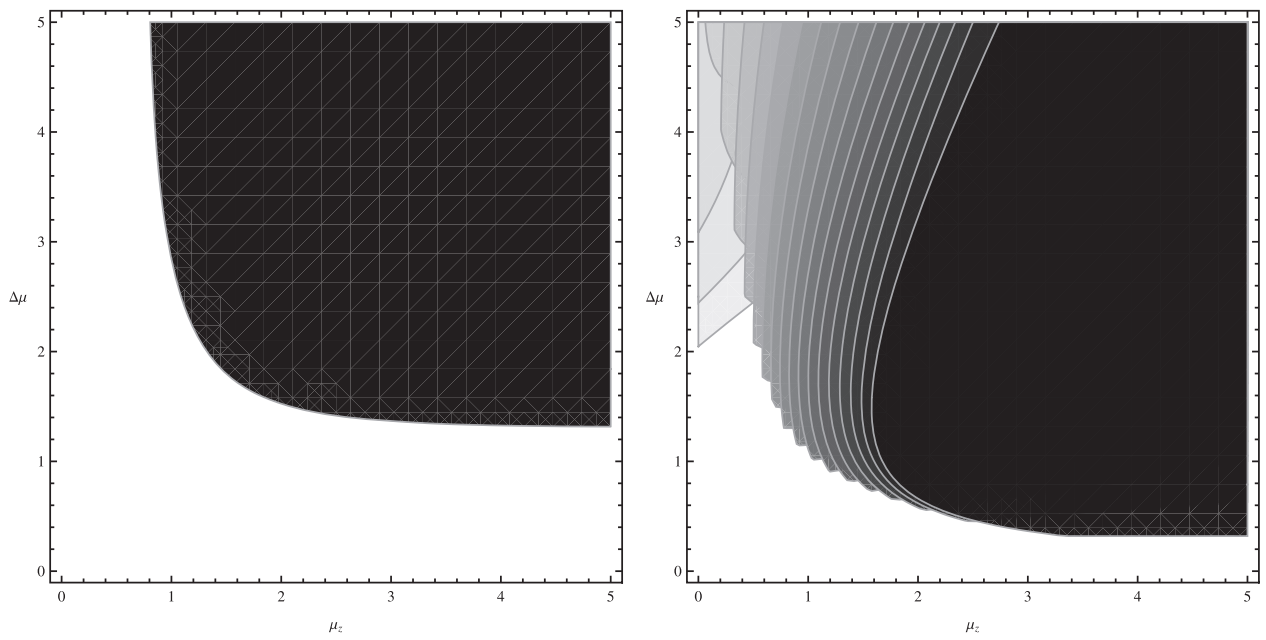

FIGURE 2

This figure depicts the loci of $\Delta \mu$ and $\mu_{z}$ leading to information complementarities. The shaded area in the left panel contains the loci for which the value of information when all agents are informed is higher than that when all agents are uninformed, that is $\left(\Delta \mu, \mu_{z}\right): \mathcal{G}(c, 1)>\mathcal{G}(c, 0)$. The shaded areas of the right panel depict the loci in markets with varying levels of informational efficiency. The loci are such that the value of information in a given market exceeds that in a slightly less informed market, that is $\left(\Delta \mu, \mu_{z}\right): \mathcal{G}(c, \lambda)>\mathcal{G}(c, \lambda-\varepsilon)$, with $\varepsilon=0.05$, and $\lambda$ ranging from $\lambda=0.05$

(lightest area) to $\lambda=1$ (darkest). The remaining parameters are $\omega_{\theta}=\omega_{\epsilon}=\omega_{z}=\tau=1$.

participating are high precisely when $\lambda$ is low-expected profits flatten in a market with many informed agents. Therefore, when $\mu_{z}$ is small, information complementarities result precisely when there are few informed agents.

\subsection{Value of price information}

More information acquisition leads to more informative prices. We now address a further issue: how valuable is the information that these prices contain? It is known that dynamic inconsistency can lead to a negative value of information both in contexts without ambiguity (e.g. Carrillo and Mariotti, 2000; and Eichberger et al.,2007) and with ambiguity (see, e.g. Siniscalchi 2011). Our focus is on information that is revealed in equilibrium. We show that, in our model, the value of information accounted for by the price can turn negative when the value of parameter uncertainty is high enough.

We denote by $\mathcal{U}_{0}(\lambda)$ the ex ante utility of a hypothetical agent whose portfolio choice is unconditional, in that it does not rely on price information 8

$$
\mathcal{U}_{0}(\lambda)=\min _{\mu \in[\underline{\mu}, \bar{\mu}]} E_{\mu}\left(-e^{-\tau x_{0} R}\right), \quad \text { where } x_{0}=\arg \max _{x}\left(\min _{\mu \in[\underline{\mu}, \bar{\mu}]} E_{\mu}\left(-e^{-\tau x R}\right)\right) .
$$

We define the value of price information as $\mathcal{G}_{p}(\lambda) \equiv-\frac{1}{\tau} \ln \left(\frac{\mathcal{U}_{U}(\lambda)}{\mathcal{U}_{0}(\lambda)}\right)$. It is the ex ante certainty equivalent gain of forming portfolio choices based on price information relative to making

8. Interestingly, $x_{0}$ can also be interpreted as the portfolio chosen by an uninformed agent in a pre-commitment equilibrium when $\lambda=1$, provided uncertainty $\Delta \mu$ is high enough (Section 6.2. 
unconditional portfolio choices. Note that the value of price information and that of parameter uncertainty in equation 4.6 add up to the value of price information for an agent who only faces risky choices at the trading stage, that is,

$$
\mathcal{G}_{p}(\lambda)=\underbrace{-\frac{1}{\tau} \ln \left(\frac{\overline{\mathcal{U}}_{U}(\lambda)}{\mathcal{U}_{0}(\lambda)}\right)}_{\text {Value of price information for interim risky trades }}-\underbrace{\left[-\frac{1}{\tau} \ln \left(\frac{\overline{\mathcal{U}}_{U}(\lambda)}{\mathcal{U}_{U}(\lambda)}\right)\right]}_{\text {Value of parameter uncertainty }},
$$

where $\overline{\mathcal{U}}_{U}(\lambda)$ is the ex ante utility in equation 4.2 .

In Appendix C, we solve for the unconditional portfolio choice in equation (5.5) (see Proposition C.1). We also show that, in the Grossman and Stiglitz 1980) model, the value of price information simply reflects the reduction of risk, thus always being positive, that is

$$
\left.\mathcal{G}_{p}^{\mathrm{gs}}(\lambda) \equiv \mathcal{G}_{p}(\lambda)\right|_{\Delta \mu=0}=\frac{1}{2 \tau} \ln \left(\frac{\operatorname{Var}(R)}{\operatorname{Var}(R \mid p)}\right)
$$

When is the value of price information negative in our model? Intuitively, equation 5.6 suggests that $\mathcal{G}_{p}(\lambda)$ is negative when the value of parameter uncertainty is high enough. Now, as the number of informed agents increases, the asset returns become risky only, not ambiguous, such that the first term on the R.H.S. of equation (5.6) approaches $\mathcal{G}_{p}^{\mathrm{gs}}(\cdot)$. Therefore, when $\lambda$ is high, not only is the value of price information lower than in Grossman-Stiglitz, but it can in fact be negative, provided the value of parameter uncertainty is high enough. The next proposition shows that, for large $\mu_{z}$, this is the case when uncertainty $\Delta \mu$ is high enough:

Proposition 4. (Negative value of price information). There exist a level of uncertainty $\overline{\Delta \mu}>0$ and an average asset supply $\bar{\mu}_{z}>0$, and a given fraction of informed agents $\bar{\lambda} \in(0,1)$, such that the value of price information is negative for all $\Delta \mu>\overline{\Delta \mu}, \mu_{z}>\bar{\mu}_{z}$, and $\lambda \in(\bar{\lambda}, 1]$.

\subsection{Multiple equilibria and the impact of an uncertainty shock}

Information complementarities can lead to multiple equilibria when information decisions are discrete choices, as in our model 9 Figure 3 illustrates instances of the value of information, $\mathcal{G}(c, \lambda)$ from equation (4.6), plotted as a function of the fraction of informed agents, $\lambda$, and obtained with four degrees of ambiguity, $\Delta \mu$.

The lower, solid and thin line is the value of information in the Grossman and Stiglitz 1980) model, that is, when $\Delta \mu=0$. As we increase $\Delta \mu$ from the benchmark value of 0 to 1 , then 1.50 and finally $2, \mathcal{G}(c, \lambda)$ increases progressively, which is consistent with Proposition 2 When $\Delta \mu=1$, there is a unique interior, and stable, equilibrium, where the fraction of informed agents is higher than in the benchmark case. As $\Delta \mu$ increases to 1.50 , the market displays two interior equilibria: the leftmost and stable equilibrium $\left(\lambda^{*}=\lambda_{S}\right)$, and the rightmost and unstable equilibrium $\left(\lambda^{*}=\right.$ $\left.\lambda_{U}\right) 10$ Note that, when $\Delta \mu=1.50$, there is a third and stable equilibrium: $\lambda^{*}=1$. The kink in

9. See Hellwig and Veldkamp 2009) and Myatt and Wallace 2012 for results regarding information complementarities and multiple equilibria under different information structures.

10. The rightmost equilibrium is unstable in that (i) for all $\lambda \in\left(\lambda_{S}, \lambda_{U}\right)$, the informed agents would be better off once uninformed, and (ii) for all $\lambda \in\left(\lambda_{U}, 1\right]$, it would be in the interest of the uninformed agents to acquire information. 


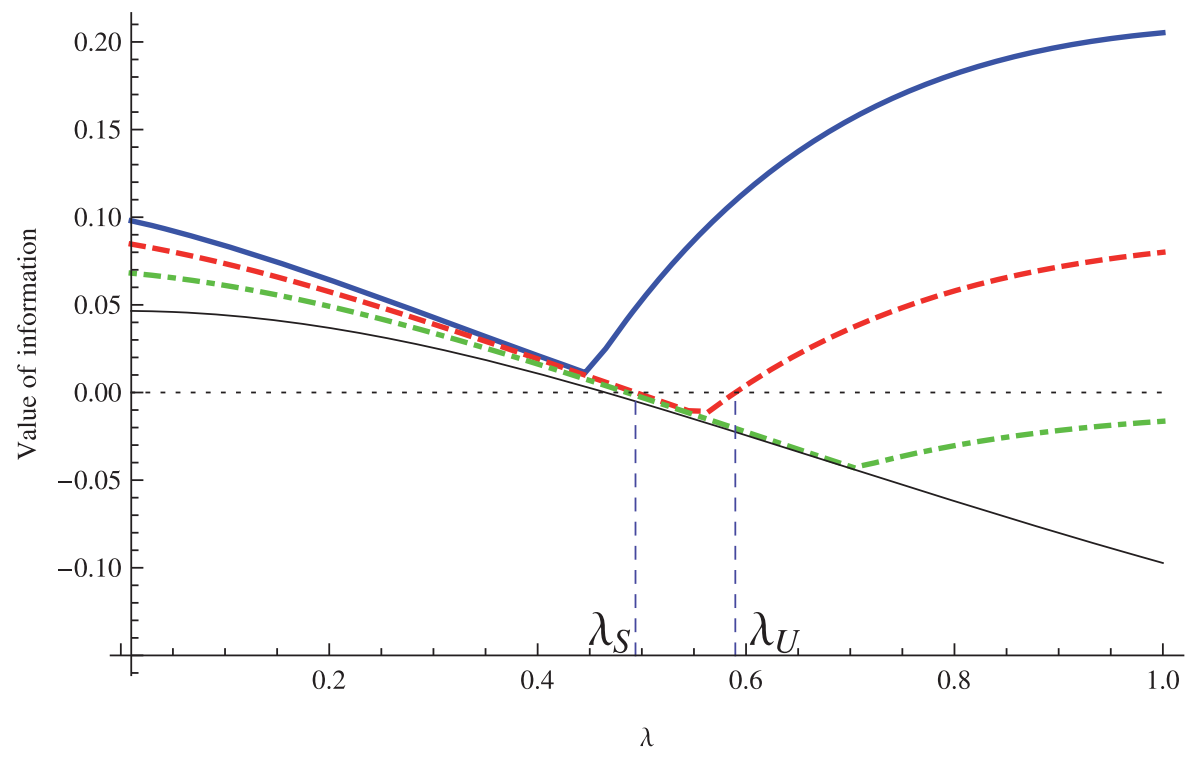

FIGURE 3

This figure depicts the value of information, $\mathcal{G}(c, \lambda)$, as a function of the fraction of informed agents, $\lambda$, for a given cost of information, $c$. The uppermost, solid and thick line is the value of information obtained with $\Delta \mu=2$. The two dashed lines below it depict the value of information when $\Delta \mu=1.50$ and $\Delta \mu=1.00$. The lower, solid and thin line depicts the value of information in the benchmark case with no ambiguity, that is, when $\Delta \mu=0$. The remaining parameter values are $\omega_{\theta}=\omega_{\epsilon}=\omega_{z}=\tau=1, \mu_{z}=2$, and $c=0.3$.

the value of information arises as the prior of the uninformed agents' shifts from $\underline{\mu}$ to $\bar{\mu}$ as $\lambda$ increases, as explained in Section 5.2(Lemmas 1 and 21 11

As $\Delta \mu$ increases in Figure 3 the value of information increases for each $\lambda$, and shifts the stable (interior) equilibria to the right, and the unstable one to the left. When $\Delta \mu$ is sufficiently high, there remains one equilibrium only, at $\lambda^{*}=1$. The uppermost, thick line, which corresponds to $\Delta \mu=2$, depicts an example of such a situation.

The right panel of Figure 4 depicts the unconditional expectation of the price as a function of $\Delta \mu$, when the proportion of agents who acquire information is determined endogenously as in the left panel. When $\Delta \mu$ is small, an increase in $\Delta \mu$ leads to a positive but modest increase in $\lambda$, and hence a lower average price, reflecting less aggressive trading of the uninformed agents. As $\Delta \mu$ becomes sufficiently large, the market shifts to its "information frenzy" regime, in which all agents become informed. The average price jumps up as a result, and then remains flat as $\Delta \mu$ increases further.

Therefore, the model predicts a non-monotonic relation between the degree of Knightian uncertainty and the average asset return. When uncertainty is low, the price falls, on average, following a series of positive changes in $\Delta \mu-$ a sequence of "uncertainty shocks". When uncertainty is sufficiently high, the price rebounds as a result of information acquisition. It jumps back to the equilibrium in the lower branch of Figure 4 when uncertainty becomes sufficiently low. Furthermore, the price exhibits path dependence and different jump sizes, according to whether

11. While these shifts in the prior are relevant to information complementarities in the context of the maxmin model of this section, they do not not appear in the smooth ambiguity extension of the model (Section 6.3, in which ambiguity aversion operates through the agents' aversion to mean-preserving spreads in expected utility values. 

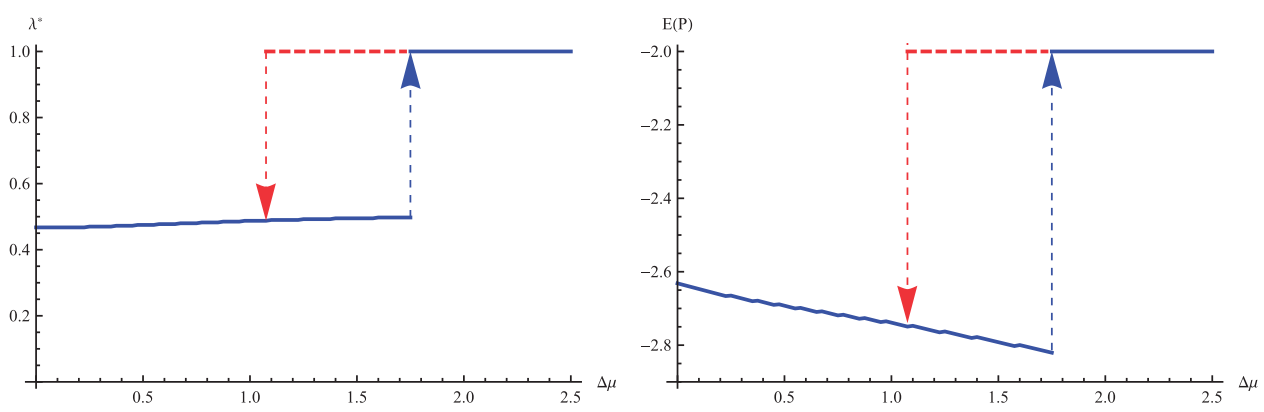

FIGURE 4

The left panel depicts the proportion of informed agents in the stable equilibria, $\lambda$, as a function of the ambiguity size, $\Delta \mu$. The right panel is the unconditional expectation of the equilibrium price corresponding to each $\lambda$ in the left panel.

The parameter values are $\omega_{\theta}=\omega_{\epsilon}=\omega_{z}=\tau=1, \mu_{z}=2$, and $c=0.3$.

uncertainty is increasing or decreasing. For all $\Delta \mu$ belonging to the area delimited by the two arrows, the average price is in the lower branch conditional on a history of positive uncertainty shocks, and in the upper branch otherwise.

Note, then, the model's prediction regarding asset price volatility. After the market has undergone an information frenzy and a highly volatile rally, the equilibrium price is, on average, in the upper branch of Figure 4. In this regime, the asset price is insensitive to further uncertainty shocks (both positive and negative) and is, on average, higher than in the lower branch's regime. Once uncertainty resettles to sufficiently low levels, the market experiences subsequent longer term "reversals", with the price undergoing the low information regime of the lower branch of Figure 4

\section{EXTENSIONS}

This section considers three extensions, aiming to analyse departures from some of the model assumptions made so far.

The first extension is learning. Full Bayesian updating is not the only learning mechanism available while dealing with ambiguity. In Section 6.1 we consider a different rule from the prior-by-prior updates of the previous sections, and discusses additional alternatives.

The second extension is commitment. The uninformed agents would be better off were they able to pre-commit to state-contingent portfolio decisions at the trading stage, as discussed in Section 4.2 In Section 6.2 we consider a model with pre-commitment.

The third extension is the separation of tastes and beliefs. Maxmin preferences do not allow us to disentangle the cognitive notion of ambiguity from the attitude towards it, as mentioned in Section 3.2 In Section 6.3 we adopt the smooth ambiguity model of Klibanoff, et al. 2005, which enables us to implement comparative statics of changing ambiguity or ambiguity aversion.

\subsection{Maximum likelihood updates of prior beliefs}

With full Bayesian updating, no learning occurs regarding the original set of priors, which means that the uninformed agents retain all their initial priors. The set of posterior beliefs on $\theta$ given the price is the set of prior beliefs on $\theta$, with each prior updated according to Bayes' rule. This section considers an updating rule based on the maximum likelihood principle, whereby the uninformed agents do not necessarily retain all of their initial priors after observing the price. 
Maximum likelihood updating was axiomatized by Gilboa and Schmeidler 1993). It captures the main flavour of classical statistical inference, as further discussed by Gilboa and Marinacci 2013).

The literature contains alternative learning approaches to those we consider in this article. A popular approach is set out by Epstein and Schneiden 2003), who consider a discrete-time extension of the multiple priors model that is recursive and, hence, dynamically consistent. I 2007, 2008) are examples of learning models within this context, in which agents have theories of multiple likelihoods that link signals to true parameters. Likewise, Hanany and Klibanoff 2007, 2009) retain dynamic consistency by allowing the update rule to depend not only on new information, but also on prior choices, in the spirit of early work by Machina (1989). In contrast, while distinct from full Bayesian updating, our analysis of this extension still considers uninformed agents who deal with preference conflicts at different decision nodes through consistent planning 12

While updating their beliefs through maximum likelihood, the uninformed agents retain those priors within their initial set that assign the highest probability to the occurrence of the observed events. That is, conditional on the observed equilibrium price having taken a value of $p$, the set of retained priors is

$$
M_{p} \equiv\left\{\mu \in[\underline{\mu}, \bar{\mu}] \mid \mu \in \arg \max _{\mu \in[\underline{\mu}, \bar{\mu}]} \operatorname{Prob}(P(\cdot)=p)\right\} .
$$

The retained priors are then updated according to Bayes' rule.

We solve the model in Online Appendix D available as Supplementary Material, where we extend the analysis of the previous sections to this new setting. In particular, we show that the equilibrium price is piecewise linear in the compound signal (Proposition D.1), that the value of information is strictly higher than in the Grossman and Stiglitz 1980) benchmark without ambiguity (Proposition D.2), and that information complementarities arise when uncertainty is high enough (Proposition D.3).

\subsection{Equilibrium with pre-commitment}

In our model, uninformed agents would be better off were they able to pre-commit to portfolio policies (Section 4.2). Moreover, the value of price information can be negative when enough agents acquire information (Section 5.3). A natural question then arises as to whether our conclusions on the value of information and learning complementarities are specific to the assumption of consistent planning. In this section, we analyse a market in which uninformed agents do pre-commit to a contingent portfolio choice $p \mapsto x_{U}(p)$, in equilibrium. (The possibility of pre-committing is not relevant to the informed agents, as shown in Online Appendix E available as Supplementary Material.) The model, solved in Online Appendix E available as Supplementary Material, leads to two main conclusions.

First, in the equilibrium with pre-commitment, the value of information is represented as in equation (4.6), with a positive value of parameter uncertainty. This value is strictly positive, unless two conditions hold simultaneously: namely, that the uninformed agents (i) optimally pre-commit to a linear portfolio policy,

$$
x_{U}(p)=\frac{E_{\mu^{*}}(f \mid p)-p}{\tau \operatorname{Var}(f \mid p)}, \text { for some constant } \mu^{*} \in[\underline{\mu}, \bar{\mu}],
$$

12. For example, see equation (D.15) in Online Appendix D available as Supplementary Material for an expression of the welfare loss that arises in this version of the model, corresponding to $\mathcal{T}(s ; \mu)$ in equation 4.5. 
and (ii) gauge their ex ante utility at the very same $\mu^{*}$. This is a fixed point problem: the prior $\hat{\mu}$ minimizing ex ante utility depends on $\mu^{*}$, and at the same time has to be equal to $\mu^{*}$ in equation 6.2) (Lemma E.2). Proposition E.1 shows that there exists a threshold $\Delta \mu^{*}$, determined in closed form (see equation (E9)), such that a fixed point exists if and only if $\Delta \mu \leq \Delta \mu^{*}$. The threshold goes to zero as $\lambda \rightarrow 1$ : the value of parameter uncertainty is always strictly positive when sufficiently many agents are informed.

Secondly, information complementarities may also arise in an equilibrium in which uninformed agents are bound to pre-commit to linear strategies. We provide conditions such that the value of information in the $\lambda=1$ case exceeds that in $\lambda=0$ case, conditions that link to the unconditional portfolio problem in Section 5.3. When $\lambda=1$, an agent who remained uninformed would pre-commit to a portfolio policy $x_{U}(p)$, which becomes less and less sensitive to the equilibrium price $p$ as $\Delta \mu$ increases, flattening out to the unconditional portfolio choice in equation 5.5) in the limit when $\Delta \mu$ is large (Lemma E.5). That is, by pre-committing, the uninformed agents mitigate concerns due to the uncertainty regarding the information contained by the price. However, by pre-committing, the uninformed agents also face much higher risk, precisely due to their policy now being unresponsive to $p$. Under conditions given by Proposition E.2, the value of information is higher in the case where $\lambda=1$ than when $\lambda=0$, reflecting the much higher risk to which the uninformed agents are exposed under higher levels of $\lambda$.

\subsection{Smooth ambiguity aversion}

Maxmin preferences do not allow us to disentangle ambiguity from ambiguity aversion. We now assume that agents display smooth ambiguity preferences, as in Klibanoff, et al. 2005) (KMM, hereafter). Given a set of priors $Q$ over $\mu$, a utility function $U$, and an increasing and concave function $h: \mathbb{R} \rightarrow \mathbb{R}$, a KMM decision maker prefers act $x^{\prime}$ to act $x$ if and only if

$$
E_{Q}\left[h\left(E_{\mu}\left(U\left(W^{x^{\prime}}\right)\right)\right)\right] \geq E_{Q}\left[h\left(E_{\mu}\left(U\left(W^{x}\right)\right)\right)\right],
$$

where $U\left(W^{x}\right)$ denotes the utility of wealth $W^{x}$ drawn from act $x$-a portfolio choice, in our context. Concavity of $h$ means that a decision maker dislikes mean-preserving spreads in expected utility values, such that he may be defined as ambiguity averse. Only if $h$ is linear will the decision maker be ambiguity neutral. One example of the function $h$ in equation 6.3 is $h(u)=$ $-\frac{1}{\sigma}\left(e^{-\sigma u}-1\right)$, where the parameter $\sigma$ is a measure of absolute ambiguity aversion. If $\sigma=0$, the model collapses to a description of a Bayesian decision maker. Maxmin expected utility obtains under the assumption that $\sigma$ is large (see Proposition 3 in KMM).

We assume that the prior $\mu \sim N\left(\mu_{0}, \omega_{\mu}\right)$, and that the function $h$ in equation (6.3) exhibits constant relative ambiguity aversion (CRAA), that is, $h(u)=-(-u)^{\alpha}$, for some constant $\alpha \geq 1$. In this setup, $\omega_{\mu}$ summarizes the extent of parameter uncertainty about $\mu$, and $\alpha$ is a measure of ambiguity aversion, with the decision maker being Bayesian if $\alpha=1$, and ambiguity averse if $\alpha>1$. The model is solved in Online Appendix F available as Supplementary Material. Its main predictions are that the value of information is higher in a market with uncertainty than in one without, and that there are complementarities in information acquisition, multiple equilibria, and possibly a negative value of price information - just as in the maxmin case.

By disentangling parameter uncertainty from aversion to it, the model allows us to single out the effect of ambiguity aversion on the value of parameter uncertainty. In Online Appendix F available as Supplementary Material, we establish that, if agents are Bayesian, that is, if $\alpha=1$, the value of parameter uncertainty is strictly positive (Lemma F.1), but it also decreases with information acquisition (Proposition F.1), such that information choices can only be strategic substitutes. Furthermore, we show that information complementarities and multiple equilibria 


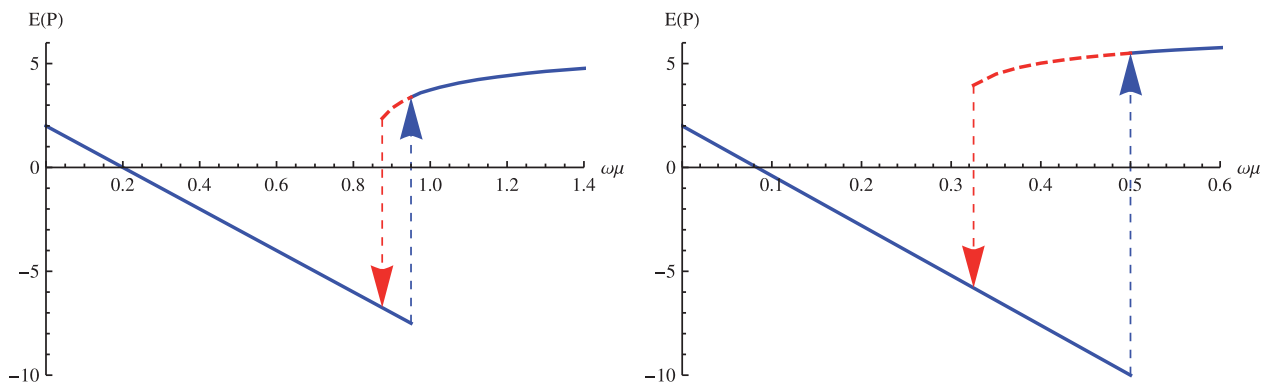

FIGURE 5

This figure depicts the unconditional expectation of the equilibrium price corresponding to the endogenous fraction of informed agents, $\lambda^{*}$, as a function of the uncertainty size, $\omega_{\mu}$, for two values of the ambiguity aversion parameter, $\alpha$. The left panel sets $\alpha=2.5$ and the right panel sets $\alpha=6$. Other parameter values are $\omega_{\theta}=\omega_{\epsilon}=\omega_{z}=\tau=1, \mu_{z}=4$,

$$
\mu_{0}=10 \text {, and } c=0.6 \text {. }
$$

(Proposition F.1), and a negative value of price information (Proposition F.2), arise only if agents are ambiguity averse, that is, if $\alpha>1$. Note that these properties arise even if, unlike in the maxmin model of Sections 35 portfolio and information choices are not based on the worstcase scenario 13 They do arise, however, through the same mechanism as that in the maxmin model - the value of parameter uncertainty increases with information acquisition.

Figure 5 depicts the comparative statics regarding the market behaviour after a change in the uncertainty aversion parameter, $\alpha$. Each panel is the counterpart to the right panel in Figure 4 and plots the average price against the uncertainty size, $\omega_{\mu}$, when the fraction of informed agents is endogenous. Similarly as in the maxmin model, the price decreases with uncertainty, on average, reflecting less aggressive trading by the uninformed agents. Once $\omega_{\mu}$ is sufficiently large, information complementarities are triggered, with an "information frenzy" that leads to a discontinuity in the average equilibrium price and then to a positive "price drift", determined by further information acquisition that occurs in a smooth fashion.

An increase in ambiguity aversion has clear implications in this market: (i) it increases the price impact of changes in uncertainty, $\omega_{\mu}$, (ii) it reduces the critical value of $\omega_{\mu}$ that triggers learning complementarities, and (iii) it leads to wider price swings. The higher price impact in (i), and the ensuing properties (ii) and (iii), are consistent with the intuition that increased ambiguity aversion leads to less aggressive asset demand. Gollien (2011) shows that this intuitive property of asset demand might not be robust to alternative specifications of $h$ in equation 6.3 , which go beyond the CRAA case of this section.

\section{CONCLUSION}

This article departs from the literature in its focus on the value of information in markets with Knightian uncertainty. How valuable is it to acquire information that other investors already have? In their seminal work, Grossman and Stiglitz 1980, p. 405) conclude that "... there is a fundamental conflict between the efficiency with which markets spread information and the incentives to acquire information". In markets with Knightian uncertainty, this conflict takes a novel form. 
In our model, more information acquisition implies that prices have more information content: as the number of informed investors grows, those who remain uninformed trade with less risk and less ambiguity. The incentives to reduce risk by acquiring information thus diminish with information acquisition, just as in Grossman and Stiglitz, but the incentives to reduce ambiguity do not. The benefit of reducing ambiguity is to allow an ambiguity averse agent to correctly interpret the information revealed by the asset prices (the "value of parameter uncertainty"). Because asset prices become increasingly informative as more investors acquire information, the value of parameter uncertainty increases with information acquisition.

When uncertainty is high enough, these ambiguity effects overwhelm those arising from risk. Information acquisition decisions become strategic complements: the more investors acquire information, the higher are the incentives to become informed. These markets then respond to even small changes in uncertainty with episodes of large price fluctuations, driven by hasty changes in information demand. Our model suggests that investors' fear of dealing with Knightian uncertainty can lead to new information-based explanations of asset market volatility.

\section{APPENDIX}

\section{A. PROOFS FOR SECTION 3}

Proof of Proposition 1. By the market-clearing condition, equation 3.6, the equilibrium price arising when the uninformed agents do not participate is:

$$
P(s)=-\frac{\tau \omega_{\epsilon}}{\lambda} \mu_{z}+\frac{\tau \omega_{\epsilon}}{\lambda} s,
$$

which is the second line in equation 3.7. Next, we compute the uninformed agents' expectation of the asset payoff, in the states of nature where these agents participate. Denote the conditional expectation $\mu_{f \mid s}(s ; \mu) \equiv E_{\mu}(f \mid S=s)$. We have,

$$
\mu_{f \mid s}(s ; \mu)=(1-\chi) \mu+\left(\frac{\lambda}{\tau \omega_{\epsilon}}\right)^{-1} \chi s, \quad \chi \equiv \frac{\left(\frac{\lambda}{\tau \omega_{\epsilon}}\right)^{2} \omega_{\theta}}{\left(\frac{\lambda}{\tau \omega_{\epsilon}}\right)^{2} \omega_{\theta}+\omega_{z}} .
$$

Using equation A.1, and $\omega_{s}=\left(\frac{\lambda}{\omega_{\epsilon} \tau}\right)^{2} \omega_{\theta}+\omega_{z}$, straightforward computations leave:

$$
\begin{aligned}
& E^{\text {buy }}(f \mid S=s)=\frac{\tau^{2} \omega_{\epsilon}^{2} \omega_{z}}{\lambda^{2} \omega_{\theta}+\tau^{2} \omega_{\epsilon}^{2} \omega_{z}} \underline{\mu}+\frac{\lambda \tau \omega_{\epsilon} \omega_{\theta}}{\lambda^{2} \omega_{\theta}+\tau^{2} \omega_{\epsilon}^{2} \omega_{z}} s \\
& E^{\text {sell }}(f \mid S=s)=\frac{\tau^{2} \omega_{\epsilon}^{2} \omega_{z}}{\lambda^{2} \omega_{\theta}+\tau^{2} \omega_{\epsilon}^{2} \omega_{z}} \bar{\mu}+\frac{\lambda \tau \omega_{\epsilon} \omega_{\theta}}{\lambda^{2} \omega_{\theta}+\tau^{2} \omega_{\epsilon}^{2} \omega_{z}} s
\end{aligned}
$$

Next, we plug equations A.2 A.3 into the demand schedule, equation 3.3, replace the result into the market-clearing condition, equation 3.6, conjecture the piece-wise linear price function in equation [3.7, and solve for undetermined coefficients, obtaining,

$$
\begin{aligned}
& \underline{a}=\frac{-\lambda^{2} \mu_{z} \tau \omega_{\epsilon} \omega_{\theta}+\left(\underline{\mu}(1-\lambda) \omega_{\epsilon}-\mu_{z} \tau \omega_{\epsilon}\left(\omega_{\epsilon}+\omega_{\theta}\right)\right) \tau^{2} \omega_{z} \omega_{\epsilon}}{\lambda^{2} \omega_{\theta}+\lambda \tau^{2} \omega_{\theta} \omega_{z} \omega_{\epsilon}+\tau^{2} \omega_{z} \omega_{\epsilon}^{2}} \\
& \bar{a}=\underline{a}+\frac{\Delta \mu(1-\lambda) \tau^{2} \omega_{z} \omega_{\epsilon}^{2}}{\lambda^{2} \omega_{\theta}+\lambda \tau^{2} \omega_{\theta} \omega_{z} \omega_{\epsilon}+\tau^{2} \omega_{z} \omega_{\epsilon}^{2}} \\
& a=-\frac{\tau \omega_{\epsilon}}{\lambda} \mu_{z} \\
& b=\frac{\left(\lambda \omega_{\theta}+\omega_{z} \tau^{2} \omega_{\epsilon}\left(\omega_{\theta}+\omega_{\epsilon}\right)\right) \tau \omega_{\epsilon}}{\lambda^{2} \omega_{\theta}+\lambda \tau^{2} \omega_{\theta} \omega_{z} \omega_{\epsilon}+\tau^{2} \omega_{z} \omega_{\epsilon}^{2}}
\end{aligned}
$$

Finally, we determine the threshold for the compound signal, $\underline{s}$ and $\bar{s}$. We use the cut-off conditions in equation 3.4 . As for $\underline{s}$, consider the first equation, $E^{\text {buy }}(f \mid P(\cdot, \cdot)=\underline{p})=\underline{p}$. For $s \leq \underline{s}$, the conjectured price function is linear in $s$. Therefore, we solve for $\underline{p}$ by equivalently solving for $\underline{s}$ in the following condition,

$$
E^{\text {buy }}(f \mid S=\underline{s})=\underline{p}=\underline{a}+b \underline{s},
$$


MELE \& SANGIORGI UNCERTAINTY AND INFORMATION INASSET MARKETS 1555

where $E^{\text {buy }}(f \mid S=\underline{s})$ is given by equation $\mathbf{A} .2$, and the second equality holds by the first line of the conjectured price function in equation 3.7. We do the same to determine $\bar{s}$, by solving,

$$
E^{\text {sell }}(f \mid S=\bar{s})=\bar{p}=\bar{a}+b \bar{s},
$$

where $E^{\text {sell }}(f \mid S=\bar{s})$ is given by equation A.3. The expressions for $\underline{s}$ and $\bar{s}$ given in Proposition 1 then follow by simple computations. Finally, we calculate the threshold prices $\bar{p}$ and $p$. We plug equations A.2 A.3 into equation 3.4, use the price function in equation 3.7], and obtain $\underline{p}=\underline{\mu}+\frac{\lambda \omega_{\theta}}{\tau \omega_{\epsilon} \omega_{z}} \mu_{z}$ and $\bar{p}=\bar{\mu}+\frac{\lambda \omega_{\theta}}{\tau \omega_{\epsilon} \omega_{z}} \mu_{z}$, which confirm that $\underline{p}<\bar{p}$.

\section{B. PROOFS FOR SECTION 4}

\section{B.1. Derivation of the ex ante utilities}

B.1.1. Informed agents. Let $\mu_{\theta \mid s}(s ; \mu)$ and $\omega_{\theta \mid s}$ denote the conditional expectation and variance of $\theta$ given $s$,

$$
E_{\mu}(\theta \mid s)=\mu_{\theta \mid s}(s ; \mu)=\mu_{f \mid s}(s ; \mu), \quad \omega_{\theta \mid s}=\frac{\omega_{z} \omega_{\theta}}{\omega_{s}},
$$

where $\mu_{f \mid s}(s ; \mu)$ is as in equation A.1. We have,

$$
E_{\mu}\left[-e^{-\tau \hat{W}_{I}}\right]=E_{\mu}\left[E\left(-e^{-\tau \hat{W}_{I}} \mid \theta, s\right)\right]=E_{\mu}\left[e^{-\tau(\mathcal{C}(\theta, s)-c)}\right],
$$

where $\hat{W}_{I}$ is the informed agent's wealth,

$$
\mathcal{C}(\theta, s) \equiv \frac{1}{2} \frac{(\theta-P(s))^{2}}{\tau \omega_{\epsilon}}
$$

is the certainty equivalent for the expected utility of the informed agent at the trading stage and, finally, $P(s)$ is the equilibrium price of Proposition 1 By conditioning upon the signal $s$ in equation B.2,

$$
E_{\mu}\left[-e^{-\tau \hat{W}_{I}}\right]=\int_{-\infty}^{\infty} E_{\mu}\left(-e^{-\tau(\mathcal{C}(\theta, \sigma)-c)} \mid \sigma\right) d \Phi\left(\sigma ; m_{s}(\mu), \omega_{s}\right)
$$

where $\Phi(\cdot ; m, \omega)$ denotes the cumulative distribution function of a normal variable with mean $m$ and variance $\omega$, and $m_{s}(\mu)$ and $\omega_{s}$ denote the mean and variance of the compound signal $s$ in equation 3.5, with

$$
m_{s}(\mu) \equiv E_{\mu}(s)=\frac{\lambda}{\tau \omega_{\epsilon}} \mu,
$$

and:

$$
E_{\mu}\left(-e^{-\tau(\mathcal{C}(\theta, \sigma)-c)} \mid \sigma\right)=e^{\tau c} \int_{-\infty}^{\infty}\left(-e^{-\tau \mathcal{C}(\theta, \sigma)}\right) d \Phi\left(\theta ; \mu_{\theta \mid s}(\sigma ; \mu), \omega_{\theta \mid s}\right)=-e^{\tau c} \sqrt{\frac{\omega_{\epsilon}}{\omega_{f \mid s}}} e^{-\tau \overline{\mathcal{C}}(\sigma ; \mu)}
$$

where

and

$$
\overline{\mathcal{C}}(s ; \mu)=\frac{1}{2} \frac{\left(E_{\mu}(\theta \mid s)-P(s)\right)^{2}}{\tau \omega_{f \mid s}},
$$

$$
\omega_{f \mid s}=\omega_{\epsilon}+\frac{\omega_{z} \omega_{\theta}}{\omega_{s}}
$$

By replacing $P(s)$ and the expression for $\mu_{\theta \mid s}(s ; \mu)$ in equation $\mathbb{B} .1$ into the expression for $\overline{\mathcal{C}}(s ; \mu)$ leaves, for $\hat{s}=\frac{1}{2}(\underline{s}+\bar{s})$,

$$
e^{-\tau \overline{\mathcal{C}}(s ; \mu)}= \begin{cases}\exp \left(-\frac{1}{2} \frac{\delta^{2}}{\omega_{f \mid s}}\left(s-\underline{s}-\frac{\hat{\delta}}{\delta} \frac{\lambda}{\tau \omega_{\epsilon}}(\mu-\underline{\mu})\right)^{2}\right), & \text { for } s<\underline{s} \\ \exp \left(-\frac{1}{2} \frac{\hat{\delta}^{2}}{\omega_{f \mid s}}\left(s-\hat{s}-\frac{\lambda}{\tau \omega_{\epsilon}} \mu\right)^{2}\right), & \text { for } s \in[\underline{s}, \bar{s}] \\ \exp \left(-\frac{1}{2} \frac{\delta^{2}}{\omega_{f \mid s}}\left(s-\bar{s}+\frac{\hat{\delta}}{\delta} \frac{\lambda}{\tau \omega_{\epsilon}}(\bar{\mu}-\mu)\right)^{2}\right), & \text { for } s>\bar{s}\end{cases}
$$

where

$$
\delta=\frac{\tau^{3} \omega_{z} \omega_{\epsilon}^{3}\left(\lambda^{2} \omega_{\theta}+\tau^{2} \omega_{z} \omega_{\epsilon}^{2}+\tau^{2} \omega_{z} \omega_{\theta} \omega_{\epsilon}\right)}{\left(\lambda^{2} \omega_{\theta}+\lambda \tau^{2} \omega_{z} \omega_{\theta} \omega_{\epsilon}+\tau^{2} \omega_{z} \omega_{\epsilon}^{2}\right)\left(\lambda^{2} \omega_{\theta}+\tau^{2} \omega_{z} \omega_{\epsilon}^{2}\right)}, \quad \hat{\delta}=\frac{\tau \omega_{\epsilon} \omega_{z}}{\lambda \omega_{s}}
$$


Finally, substituting equations B.5 and B.7 into equation B.3, and integrating, leaves the following closed-form expression for the expectation in equation 4.1 of the main text,

$$
E_{\mu}\left[-e^{-\tau(\mathcal{C}(\theta, \sigma)-c)}\right]=e^{\tau c} \sqrt{\frac{\omega_{\epsilon}}{\omega_{f \mid s}}} \cdot \sum_{\ell \in\{\text { buy,np,sell }\}} I_{\mu}^{\ell},
$$

where, denoting $\Phi(\cdot) \equiv \Phi(\cdot ; 0,1)$,

$$
\begin{aligned}
I_{\mu}^{\text {buy }} & =-\kappa \exp \left(-\frac{\delta^{2}\left(\frac{\omega_{s}}{\omega_{z}} \mu_{z}+\gamma_{0}(\mu-\underline{\mu})\right)^{2}}{2\left(\omega_{f \mid s}+\delta^{2} \omega_{s}\right)}\right) \Phi\left(\frac{\kappa}{\sqrt{\omega_{s}}}\left(\frac{\omega_{s}}{\omega_{z}} \mu_{z}-\gamma_{1}(\mu-\underline{\mu})\right)\right) \\
I_{\mu}^{\mathrm{np}} & =-\hat{\kappa} \exp \left(-\frac{\hat{\delta}^{2}\left(\frac{\omega_{s}}{\omega_{z}} \mu_{z}\right)^{2}}{2\left(\omega_{f \mid s}+\hat{\delta}^{2} \omega_{s}\right)}\right) \\
& \times\left[\Phi\left(\frac{\hat{\kappa}}{\sqrt{\omega_{s}}}\left(\frac{\omega_{s}}{\omega_{z}} \mu_{z}+\gamma_{2}(\bar{\mu}-\mu)\right)\right)-\Phi\left(\frac{\hat{\kappa}}{\sqrt{\omega_{s}}}\left(\frac{\omega_{s}}{\omega_{z}} \mu_{z}-\gamma_{2}(\mu-\underline{\mu})\right)\right)\right] \\
I_{\mu}^{\text {sell }} & =-\kappa \exp \left(-\frac{\left.\delta^{2}\left(\frac{\omega_{s}}{\omega_{z}} \mu_{z}-\gamma_{0}(\bar{\mu}-\mu)\right)^{2}\right)}{2\left(\omega_{f \mid s}+\delta^{2} \omega_{s}\right)}\right)\left[1-\Phi\left(\frac{\kappa}{\sqrt{\omega_{s}}}\left(\frac{\omega_{s}}{\omega_{z}} \mu_{z}+\gamma_{1}(\bar{\mu}-\mu)\right)\right)\right]
\end{aligned}
$$

and:

$$
\begin{gathered}
\kappa=\sqrt{\frac{\omega_{f \mid s}}{\omega_{f \mid s}+\delta^{2} \omega_{s}}}, \hat{\kappa}=\sqrt{\frac{\omega_{f \mid s}}{\omega_{f \mid s}+\hat{\delta}^{2} \omega_{s}}}, \\
\gamma_{0}=\left(\frac{\hat{\delta}}{\delta}-1\right) \frac{\lambda}{\tau \omega_{\epsilon}}, \gamma_{1}=\left(1+\delta \frac{\hat{\delta} \omega_{s}}{\omega_{f \mid s}}\right) \frac{\lambda}{\tau \omega_{\epsilon}}, \gamma_{2}=\left(1+\hat{\delta} \frac{\hat{\delta} \omega_{s}}{\omega_{f \mid s}}\right) \frac{\lambda}{\tau \omega_{\epsilon}} .
\end{gathered}
$$

B.1.2. Uninformed agents. We derive the expression for $\mathcal{T}$ in equation 4.5, and a closed-form expression for the expectation in the second equality of equation 4.4. We have:

$$
\begin{aligned}
E_{\mu}\left[-e^{-\tau \hat{W}_{U}} \mid s\right] & =E_{\mu}\left[-e^{-\tau x_{U}(s)(f-P(s))} \mid s\right] \\
& =-\exp \left(-\tau x_{U}(s)\left(\mu_{f \mid s}(s ; \mu)-P(s)\right)+\frac{\tau^{2}}{2} x_{U}^{2}(s) \omega_{f \mid s}\right),
\end{aligned}
$$

where $\hat{W}_{U}$ is the uninformed agent's wealth,

$$
x_{U}(s)=\frac{\mu_{f \mid s}\left(s ; \mu^{*}(s)\right)-P(s)}{\tau \omega_{f \mid s}},
$$

and $\mu^{*}(s)$ is defined as in second of equation 4.3 in the main text. Replacing the previous expression for $x_{U}(s)$ into equation B.10 leads to the second equality of equations 4.4, where:

$$
\mathcal{T}(s ; \mu)= \begin{cases}\frac{(\underline{\mu}-\mu)^{2}(1-\chi)^{2}}{2 \tau \omega_{f \mid s}}, & \text { for } s<\underline{s} \\ \overline{\mathcal{C}}(s ; \mu), & \text { for } s \in[\underline{s}, \bar{s}] \\ \frac{(\bar{\mu}-\mu)^{2}(1-\chi)^{2}}{2 \tau \omega_{f \mid s}}, & \text { for } s>\bar{s}\end{cases}
$$

and $\chi$ is defined as in equation A.1. Finally, we use equations B.7 and B.11 to determine the expectation in the ex ante utility for the uninformed in equations 4.4,

$$
E_{\mu}\left[-e^{-\tau(\overline{\mathcal{C}}(s ; \mu)-\mathcal{T}(s ; \mu))}\right]=\sum_{\ell \in\{\text { buy,np, sell }\}} J_{\mu}^{\ell},
$$


where

$$
\begin{aligned}
& J_{\mu}^{\text {buy }}=\exp \left(\frac{(\underline{\mu}-\mu)^{2}(1-\chi)^{2}}{2 \omega_{f \mid s}}\right) \cdot I_{\mu}^{\text {buy }}, \\
& J_{\mu}^{\mathrm{np}}=-\left[\Phi\left(\frac{\bar{s}-m_{s}(\mu)}{\sqrt{\omega_{s}}}\right)-\Phi\left(\frac{\underline{s}-m_{s}(\mu)}{\sqrt{\omega_{s}}}\right)\right] \\
& J_{\mu}^{\text {sell }}=\exp \left(\frac{(\bar{\mu}-\mu)^{2}(1-\chi)^{2}}{2 \omega_{f \mid s}}\right) \cdot \cdot_{\mu}^{\text {sell }},
\end{aligned}
$$

and $I_{\mu}^{\text {buy }}$ and $I_{\mu}^{\text {sell }}$ are the same as in equation $\underline{\text { B.9. }}$.

\section{B.2. State contingent plans dominating consistent planning}

We show that $x_{U}(s) \equiv x_{U}(p, P(\theta, z))$ in equation 3.3 is dominated by alternative portfolio policies, in that the latter deliver a higher $e x$ ante utility than $\mathcal{U}_{U}(\lambda)$ in equation 4.4. We aim to tilt $x_{U}(\cdot)$ by a function such that the resulting tilted asset demand schedule generates a higher ex ante utility.

Consider the following alternative contingent portfolio rule,

$$
\hat{x}(s, t)=\frac{\mu_{f \mid s}\left(s ; \mu^{*}(s)+t h(s)\right)-P(s)}{\tau \omega_{f \mid s}},
$$

where $\mu_{f \mid s}(s ; \mu)$ is defined as in equation A.1, $t \geq 0$ and $h(s)$ is any continuous function such that: (i) $h(s) \geq 0$ for $s \leq \underline{s}$, (ii) $h(s)=0$ for $s \in[\underline{s}, \bar{s}]$, and (iii) $h(s) \leq 0$ for $s \geq \underline{s}$, with $h(s)$ being strictly different from zero on some open set for both $s \leq \underline{s}$ and $s \geq \bar{s}$. Note that by construction,

$$
x_{U}(s)=\hat{x}(s, 0) \text { for all } s .
$$

Denote with $\hat{\mathcal{U}}(t)$ the ex ante utility corresponding to $\hat{x}(\cdot, t)$. Define

$$
f(\mu, t)=E_{\mu}\left[-e^{-\frac{\left(\mu_{f \mid s}(s ; \mu)-P(s)\right)^{2}}{2 \omega_{f \mid s}}+\frac{\left(\mu^{*}(s)+t h(s)-\mu\right)^{2}(1-\chi)^{2}}{2 \omega_{f \mid s}}}\right] .
$$

Using the definition of $\mu^{*}(\cdot)$ in equation 4.3, and that of $h(\cdot)$, we obtain:

$$
\begin{aligned}
\left.\frac{\partial f(\mu, t)}{\partial t}\right|_{t=0} & =\int_{-\infty}^{\underline{s}} e^{-\frac{\left(\mu_{f \mid s}(s ; \mu)-P(s)\right)^{2}}{2 \omega_{f \mid s}}+\frac{(\mu-\underline{\mu})^{2}(1-\chi)^{2}}{2 \omega_{f \mid s}}}\left(\frac{(\mu-\underline{\mu})(1-\chi)^{2}}{\omega_{f \mid s}} h(s)\right) \phi\left(s ; \frac{\lambda}{\tau \omega_{\epsilon}} \mu, \omega_{s}\right) d s \\
& +\int_{\bar{s}}^{\infty} e^{-\frac{\left(\mu_{f \mid s}(s ; \mu)-P(s)\right)^{2}}{2 \omega_{f \mid s}}+\frac{(\mu-\bar{\mu})^{2}(1-\chi)^{2}}{2 \omega_{f \mid s}}}\left(\frac{(\mu-\bar{\mu})(1-\chi)^{2}}{\omega_{f \mid s}} h(s)\right) \phi\left(s ; \frac{\lambda}{\tau \omega_{\epsilon}} \mu, \omega_{s}\right) d s,
\end{aligned}
$$

where $\phi$ denotes the normal density, $d \Phi \equiv \phi d s$. Given that $\mu-\mu \geq 0$ and and $h(s) \geq 0$ for $s \leq \underline{s}$, with the inequality being strict on some open set, the first term is strictly positive for all $\mu>\underline{\mu}$ and zero for $\mu=\mu$. Given that $\mu-\bar{\mu} \leq 0$ and $h(s) \leq 0$ for $s \geq \bar{s}$, with the inequality being strict on some open set, the second term is strictly positive for all $\mu<\bar{\mu}$ and zero for $\mu=\bar{\mu}$. Therefore, $\left.\frac{\partial f(\mu, t)}{\partial t}\right|_{t=0}>0$ for all $\mu$, which implies that there exist sufficiently small values of $t>0$ such that $f(\mu, t)>f(\mu, 0)$ for all $\mu$, and, then, $\hat{\mathcal{U}}(t)=\min _{\mu \in[\mu, \bar{\mu}]} f(\mu, t)>\min _{\mu \in[\mu, \bar{\mu}]} f(\mu, 0)$. But by equation $\left[\mathbf{B} .14, \hat{\mathcal{U}}(0)=\mathcal{U}_{U}\right.$. It follows that for $t$ small enough, $\hat{\mathcal{U}}(t)>\mathcal{U}_{U}$.

Proof of Proposition 2. Consider the definition of the value of information for any $\lambda$ in equation 4.6 . We wish to show that for $\Delta \mu>0$,

$$
\frac{E_{\mu_{U}}\left[-e^{-\tau\left(\overline{\mathcal{C}}\left(s ; \mu_{U}\right)-\mathcal{T}\left(s ; \mu_{U}\right)\right)}\right]}{E_{\mu_{I}}\left[-e^{-\tau \overline{\mathcal{C}}\left(s ; \mu_{I}\right)}\right]}>1 .
$$

Because $E_{\mu_{I}}\left[-e^{-\tau \overline{\mathcal{C}}\left(s ; \mu_{I}\right)}\right]$ and $E_{\mu_{U}}\left[-e^{-\tau\left(\overline{\mathcal{C}}\left(s ; \mu_{U}\right)-\mathcal{T}\left(s ; \mu_{U}\right)\right)}\right]$ are both strictly negative, the previous inequality holds true if:

$$
E_{\mu_{I}}\left[-e^{-\tau \overline{\mathcal{C}}\left(s ; \mu_{I}\right)}\right]>E_{\mu_{U}}\left[-e^{-\tau\left(\overline{\mathcal{C}}\left(s ; \mu_{U}\right)-\mathcal{T}\left(s ; \mu_{U}\right)\right)}\right]
$$

where we define, as in the main text:

$$
\mu_{I} \in \arg \min _{\mu \in[\underline{\mu}, \bar{\mu}]} E_{\mu}\left[-e^{-\tau \overline{\mathcal{C}}(s ; \mu)}\right], \quad \mu_{U} \in \arg \min _{\mu \in[\underline{\mu}, \bar{\mu}]} E_{\mu}\left[-e^{-\tau(\overline{\mathcal{C}}(s ; \mu)-\mathcal{T}(s ; \mu))}\right] .
$$


To show that the inequality B.15 is true, suppose the contrary, i.e. that:

$$
E_{\mu_{I}}\left[-e^{-\tau \overline{\mathcal{C}}\left(s ; \mu_{I}\right)}\right] \leq E_{\mu_{U}}\left[-e^{-\tau\left(\overline{\mathcal{C}}\left(s ; \mu_{U}\right)-\mathcal{T}\left(s ; \mu_{U}\right)\right)}\right] .
$$

Because $\mathcal{T}(s ; \mu)$ is non-negative and strictly positive on an open set of values of $s$ (equation B.11), we have that,

$$
E_{\mu_{I}}\left[-e^{-\tau \overline{\mathcal{C}}\left(s ; \mu_{I}\right)}\right]>E_{\mu_{I}}\left[-e^{-\tau\left(\overline{\mathcal{C}}\left(s ; \mu_{I}\right)-\mathcal{T}\left(s ; \mu_{I}\right)\right)}\right] .
$$

Combining B.16 with B.17 yields,

$$
E_{\mu_{I}}\left[-e^{-\tau\left(\overline{\mathcal{C}}\left(s ; \mu_{I}\right)-\mathcal{T}\left(s ; \mu_{I}\right)\right)}\right]<E_{\mu_{I}}\left[-e^{-\tau \overline{\mathcal{C}}\left(s ; \mu_{I}\right)}\right] \leq E_{\mu_{U}}\left[-e^{-\tau\left(\overline{\mathcal{C}}\left(s ; \mu_{U}\right)-\mathcal{T}\left(s ; \mu_{U}\right)\right)}\right],
$$

contradicting that $\mu_{U}$ minimizes $E_{\mu}\left[-e^{-\tau(\overline{\mathcal{C}}(s ; \mu)-\mathcal{T}(s ; \mu))}\right]$.

The next corollary follows directly by Proposition 2

Corollary B.1. Information is purchased by more agents in the presence of ambiguity than in markets without ambiguity, and strictly so unless the equilibrium fraction of informed agents in both markets is either zero or one.

Proof. Let $\lambda^{*}(\Delta \mu) \in(0,1]$ solve the indifference condition, $\mathcal{G}(c, \lambda)=0$. Assume now that $\lambda^{*}(0) \geq \lambda^{*}(\Delta \mu)$, for some $\Delta \mu>0$. By Proposition 2 and the fact that the first term in the R.H.S. of equation 4.6 is decreasing in $\lambda$, this cannot be the case as we would have, $\mathcal{G}\left(c, \lambda^{*}(\Delta \mu)\right)>0$. Furthermore, since $\left.\mathcal{G}(c, \lambda)\right|_{\Delta \mu>0}>\left.\mathcal{G}(c, \lambda)\right|_{\Delta \mu=0}$ for all $\lambda$ by Proposition 2 then $\lambda^{*}(\Delta \mu)=0$ implies $\lambda^{*}(0)=0$, and $\lambda^{*}(0)=1$ implies $\lambda^{*}(\Delta \mu)=1$.

\section{PROOFS FOR SECTION 5}

We prove Proposition 3 by relying on Lemmas 1 and 2 in the main text. We prove these two lemmas first.

Proof of Lemma 1. We first show that for $\lambda=0$, there exists a $\hat{\mu}_{z}>0$, such that for all $\mu_{z} \geq \hat{\mu}_{z}$, the ex ante utility of the informed agents occurs at $\mu=\underline{\mu}$, i.e. $\mathcal{U}_{I}(c, 0)=E_{\underline{\mu}}\left[-e^{-\tau \hat{W}_{I}}\right]$. Note that because $I_{\mu}^{\mathrm{np}}=0$ when $\lambda=0$, it is sufficient to show that for all $\mu_{z} \geq \hat{\mu}_{z}, \arg \min _{\mu \in[\underline{\mu}, \bar{\mu}]}\left(I_{\mu}^{\text {buy }}+I_{\mu}^{\text {sell }}\right)=\underline{\mu}$. When $\lambda=0$, by equation B.9 we have,

$$
\begin{aligned}
& I_{\mu}^{\text {buy }}=-c_{0} \exp \left(-\frac{c_{0}^{2}}{2} \frac{\left(\tau \omega_{f} \mu_{z}+(\mu-\underline{\mu})\right)^{2}}{\omega_{f}}\right) \Phi\left(\frac{c_{0}}{\sqrt{\omega_{z}}}\left(\mu_{z}-\tau \omega_{z}(\mu-\underline{\mu})\right)\right) \\
& I_{\mu}^{\text {sell }}=-c_{0} \exp \left(-\frac{c_{0}^{2}}{2} \frac{\left(\tau \omega_{f} \mu_{z}-(\bar{\mu}-\mu)\right)^{2}}{\omega_{f}}\right)\left[1-\Phi\left(\frac{c_{0}}{\sqrt{\omega_{z}}}\left(\mu_{z}+\tau \omega_{z}(\bar{\mu}-\mu)\right)\right)\right]
\end{aligned}
$$

where $\omega_{f}=\omega_{\theta}+\omega_{\epsilon}$, and $c_{0}=\left(1+\tau^{2} \omega_{f} \omega_{z}\right)^{-\frac{1}{2}}$.

We show that $\mu \mapsto\left(I_{\mu}^{\text {buy }}+I_{\mu}^{\text {sell }}\right)$ is increasing when $\mu_{z}$ is sufficiently large. We have,

$$
\begin{aligned}
c_{0}^{-1} \frac{\partial}{\partial \mu} I_{\mu}^{\text {buy }} & =e^{-\frac{c_{0}^{2}}{2} \frac{\left(\tau \omega_{f} \mu_{z}+(\mu-\underline{\mu})\right)^{2}}{\omega_{f}}}\left(c_{0}^{2} \frac{\tau \omega_{f} \mu_{z}+(\mu-\underline{\mu})}{\omega_{f}}\right) \Phi\left(\frac{c_{0}}{\sqrt{\omega_{z}}}\left(\mu_{z}-\tau \omega_{z}(\mu-\underline{\mu})\right)\right) \\
& +e^{-\frac{c_{0}^{2}}{2} \frac{\left(\tau \omega_{f} \mu_{z}+(\mu-\underline{\mu})\right)^{2}}{\omega_{f}}} \phi\left(\frac{c_{0}}{\sqrt{\omega_{z}}}\left(\mu_{z}-\tau \omega_{z}(\mu-\underline{\mu})\right)\right) c_{0} \tau \sqrt{\omega_{z}},
\end{aligned}
$$

and,

$$
\begin{aligned}
c_{0}^{-1} \frac{\partial}{\partial \mu} I_{\mu}^{\text {sell }} & =e^{-\frac{c_{0}^{2}}{2} \frac{\left(\tau \omega_{f} \mu_{z}-(\bar{\mu}-\mu)\right)^{2}}{\omega_{f}}} c_{0}^{2} \frac{\tau \omega_{f} \mu_{z}-(\bar{\mu}-\mu)}{\omega_{f}}\left[1-\Phi\left(\frac{c_{0}\left(\mu_{z}+\tau \omega_{z}(\bar{\mu}-\mu)\right)}{\sqrt{\omega_{z}}}\right)\right] \\
& -e^{-\frac{c_{0}^{2}}{2} \frac{\left(\tau \omega_{f} \mu_{z}-(\bar{\mu}-\mu)\right)^{2}}{\omega_{f}}} \phi\left(\frac{c_{0}}{\sqrt{\omega_{z}}}\left(\mu_{z}+\tau \omega_{z}(\bar{\mu}-\mu)\right)\right) c_{0} \tau \sqrt{\omega_{z}} \\
& \equiv B_{1}^{\text {sell }}+B_{2}^{\text {sell }} .
\end{aligned}
$$


The term $B_{1}^{\text {sell }}$ is strictly positive for all $\mu$ whenever $\mu_{z}>\mu_{z}^{*} \equiv \frac{\Delta \mu}{\tau \omega_{f}}$. After tedious but straightforward computations, we find that

$$
c_{0}^{-1} \frac{\partial}{\partial \mu} I_{\mu}^{\text {buy }}+B_{2}^{\text {sell }}=\exp \left(-\frac{c_{0}^{2}}{2} \frac{\left(\tau \omega_{f} \mu_{z}+(\mu-\underline{\mu})\right)^{2}}{\omega_{f}}\right) \cdot\left(H_{1}(\mu)+H_{2}(\mu)\right),
$$

where,

$$
\begin{aligned}
H_{1}(\mu) & \equiv\left(c_{0}^{2} \frac{\tau \omega_{f} \mu_{z}+(\mu-\underline{\mu})}{\omega_{f}}\right) \Phi\left(\frac{c_{0}}{\sqrt{\omega_{z}}}\left(\mu_{z}-\tau \omega_{z}(\mu-\underline{\mu})\right)\right) \\
H_{2}(\mu) & \equiv \phi\left(\frac{c_{0}}{\sqrt{\omega_{z}}}\left(\mu_{z}+\tau \omega_{z}(\bar{\mu}-\mu)\right)\right) \exp \left(c_{0}^{2}\left(\mu_{z} \tau-\mu \tau^{2} \omega_{z}\right) \Delta \mu\right) \\
& \times\left[1-\exp \left(\frac{c_{0}^{2} \Delta \mu\left(1+\tau^{2} \omega_{z} \omega_{f}\right)}{\omega_{f}} \mu\right)\right] c_{0} \tau \sqrt{\omega_{z}} .
\end{aligned}
$$

The function $H_{1}(\mu)$ is clearly positive for all $\mu$ whenever $\mu_{z}>\mu_{z}^{*}$. The function $H_{2}(\mu)$ is zero for $\mu=0$, positive for $\mu<0$, and negative for $\mu>0$. Therefore, the function $H(\mu) \equiv H_{1}(\mu)+H_{2}(\mu)$ is positive for all $\mu \leq 0$. If, instead, $\mu>0$, we have, clearly, that $\min _{\mu \in(0, \bar{\mu}]} H_{2}(\mu)=H_{2}\left(\mu_{*}\right) \equiv \bar{H}_{2}>-\infty$, for some $\mu_{*} \in(0, \bar{\mu}]$ and $\lim _{\mu_{z} \rightarrow \infty} H_{2}(\mu)=0$ for all $\mu \in[\underline{\mu}, \bar{\mu}]$. Moreover, for each $\mu$, the function $H_{1}(\mu)$ is increasing in $\mu_{z}$, with $\lim _{\mu_{z} \rightarrow \infty} H_{1}(\mu)=\infty$. Therefore, for each $\mu>0$, there exists a finite $\hat{\mu}_{z}$, such that $H(\mu) \geq H_{1}(\mu)+\bar{H}_{2}>0$ for all $\mu_{z} \geq \hat{\mu}_{z}$. Take $\tilde{\mu}_{z}=\max \left\{\mu_{z}^{*}, \hat{\mu}_{z}\right\}$ to conclude about the informed agents' choice.

Next, we show that for $\lambda=0$, there exists a $\check{\mu}_{z}>0$, such that for all $\mu_{z} \geq \check{\mu}_{z}$, the ex ante utility of the uninformed agents also occurs at $\mu=\underline{\mu}$, i.e. $\mathcal{U}_{U}(0)=E_{\underline{\mu}}\left[-e^{-\tau \hat{W}_{U}}\right]$. When $\lambda=0$, we have, by equation B.12], that,

$$
J_{\mu}^{\text {buy }}+J_{\mu}^{\text {sell }}=\exp \left(\frac{(\underline{\mu}-\mu)^{2}}{2 \omega_{f}}\right) \cdot I_{\mu}^{\text {buy }}+\exp \left(\frac{(\bar{\mu}-\mu)^{2}}{2 \omega_{f}}\right) \cdot I_{\mu}^{\text {sell }}
$$

where $I_{\mu}^{\text {buy }}$ and $I_{\mu}^{\text {sell }}$ are as in equations C.2 - C.3. We need to show that $A_{1}+A_{2}>0$ for all $\mu$, where,

$$
A_{1} \equiv c_{0}^{-1} \frac{\partial}{\partial \mu}\left(\exp \left(\frac{(\underline{\mu}-\mu)^{2}}{2 \omega_{f}}\right) \cdot I_{\mu}^{\text {buy }}\right)=e^{\frac{(\underline{\mu}-\mu)^{2}}{2 \omega_{f}}} \cdot\left(\frac{\underline{\mu}-\mu}{\omega_{f}}\left(-c_{0}^{-1} I_{\mu}^{\text {buy }}\right)+c_{0}^{-1} \frac{\partial}{\partial \mu} I_{\mu}^{\text {buy }}\right),
$$

and,

$$
A_{2} \equiv c_{0}^{-1} \frac{\partial}{\partial \mu}\left(\exp \left(\frac{(\bar{\mu}-\mu)^{2}}{2 \omega_{f}}\right) \cdot I_{\mu}^{\text {sell }}\right)=e^{\frac{(\bar{\mu}-\mu)^{2}}{2 \omega_{f}}} \cdot\left(\frac{\bar{\mu}-\mu}{\omega_{f}}\left(-c_{0}^{-1} I_{\mu}^{\text {sell }}\right)+c_{0}^{-1} \frac{\partial}{\partial \mu} I_{\mu}^{\text {sell }}\right),
$$

and the terms, $\frac{\partial}{\partial \mu} I_{\mu}^{\text {buy }}$ and $\frac{\partial}{\partial \mu} I_{\mu}^{\text {sell }}$, are as in equations C.3 C.4. By equations C.1 and C.3.

$$
\begin{aligned}
& \frac{\underline{\mu}-\mu}{\omega_{f}}\left(-c_{0}^{-1} I_{\mu}^{\text {buy }}\right)+c_{0}^{-1} \frac{\partial}{\partial \mu} I_{\mu}^{\text {buy }} \\
= & \left(c_{0}^{2} \frac{\tau \omega_{f} \mu_{z}+(\mu-\underline{\mu})}{\omega_{f}}-\frac{\mu-\underline{\mu}}{\omega_{f}}\right) \exp \left(-\frac{c_{0}^{2}}{2} \frac{\left(\tau \omega_{f} \mu_{z}+(\mu-\underline{\mu})\right)^{2}}{\omega_{f}}\right) \Phi\left(\frac{c_{0}}{\sqrt{\omega_{z}}}\left(\mu_{z}-\tau \omega_{z}(\mu-\underline{\mu})\right)\right) \\
& +\exp \left(-\frac{c_{0}^{2}}{2} \frac{\left(\tau \omega_{f} \mu_{z}+(\mu-\underline{\mu})\right)^{2}}{\omega_{f}}\right) \phi\left(\frac{c_{0}}{\sqrt{\omega_{z}}}\left(\mu_{z}-\tau \omega_{z}(\mu-\underline{\mu})\right)\right) c_{0} \tau \sqrt{\omega_{z}} \\
\equiv & A_{11}+A_{12},
\end{aligned}
$$

such that $A_{11}>0$ for all $\mu$ whenever $\mu_{z}>\mu_{z}^{* *} \equiv \frac{\Delta \mu}{c_{0}^{2} \tau \omega_{f}}\left(1-c_{0}^{2}\right)=\Delta \mu \tau \omega_{z}$. Next, we show that for $\mu_{z}$ large enough, $A_{3} \equiv$ $\exp \left(\frac{(\underline{\mu}-\mu)^{2}}{2 \omega_{f}}\right) A_{12}+A_{2}>0$, for all $\mu$, thereby completing the proof. We use equations C.2 and C.4, and use the 
resulting expressions for $A_{2}$ to obtain, after tedious but straightforward computations,

$$
\begin{aligned}
A_{3} & =\exp \left(\frac{(\underline{\mu}-\mu)^{2}}{2 \omega_{f}}\right) \exp \left(-\frac{c_{0}^{2}}{2} \frac{\left(\tau \omega_{f} \mu_{z}+(\mu-\underline{\mu})\right)^{2}}{\omega_{f}}\right) \phi\left(\frac{c_{0}}{\sqrt{\omega_{z}}}\left(\mu_{z}-\tau \omega_{z}(\mu-\underline{\mu})\right)\right) \\
& \times \frac{c_{0} \tau \sqrt{\omega_{z}}}{\sqrt{2 \pi}}\left(1-\exp \left(-A_{31} \cdot \Delta \mu\right)\right) \\
& +\exp \left(\frac{(\bar{\mu}-\mu)^{2}}{2 \omega_{f}}\right) \exp \left(-\frac{c_{0}^{2}}{2} \frac{\left(\tau \omega_{f} \mu_{z}-(\bar{\mu}-\mu)\right)^{2}}{\omega_{f}}\right) \\
& \times\left[\left(\frac{\bar{\mu}-\mu}{\omega_{f}}+c_{0}^{2} \frac{\tau \omega_{f} \mu_{z}-(\bar{\mu}-\mu)}{\omega_{f}}\right)\left(1-\Phi\left(\frac{c_{0}\left(\mu_{z}+\tau \omega_{z}(\bar{\mu}-\mu)\right)}{\sqrt{\omega_{z}}}\right)\right)\right] \\
& \equiv A_{32}+A_{33}
\end{aligned}
$$

and,

$$
A_{31} \equiv c_{0} \tau\left(c_{0}+\sqrt{\omega_{z}}\right) \mu_{z}+\left(\omega_{f}^{-1}+c_{0}^{2} \omega_{f}^{-1}-\tau^{2} \omega_{z}^{\frac{3}{2}} c_{0}\right) \mu
$$

We have that (i) $A_{33}>0$ for all $\mu$ whenever $\mu_{z}>\mu_{z}^{*}=\frac{\Delta \mu}{\tau \omega_{f}}$, and (ii) for all $\mu, A_{32}>0 \Longleftrightarrow A_{31}>0$. Inspection of the definition of $A_{31}$ reveals that there exists a $\mu_{z}^{* * *}>0$ such that $A_{31}>0$ for all $\mu$ whenever $\mu_{z}>\mu_{z}^{* * *}$. Now take $\check{\mu}_{z}=$ $\max \left\{\mu_{z}^{*}, \mu_{z}^{* *}, \mu_{z}^{* * *}\right\}$ to conclude about the uninformed agents' choice.

Proof of Lemma 2. We first show that for $\lambda=1$, the ex ante utility of the informed agents is independent of $\mu$. By a direct calculation,

$$
\mathcal{I}_{1} \equiv \lim _{\lambda \rightarrow 1} \sum_{\ell \in\{\text { buy,np,sell }\}} I_{\mu}^{\ell}=-c_{1} \sqrt{\frac{\omega_{\theta}+\tau^{2} \omega_{z} \omega_{\epsilon} \omega_{f}}{c_{2}}} \exp \left(-\frac{1}{2} \tau^{2} \omega_{\epsilon} c_{1}^{2} \mu_{z}^{2}\right),
$$

where $c_{1}=\left(1+\tau^{2} \omega_{z} \omega_{\epsilon}\right)^{-\frac{1}{2}}$ and $c_{2}=\tau^{2} \omega_{z} \omega_{\epsilon}^{2}+\omega_{\theta}$, which is independent of $\mu$, as claimed.

Next, we show that there exist a $\breve{\mu}_{z}>0$ and a $\bar{\lambda} \in(0,1)$ such that for all $\mu_{z} \geq \breve{\mu}_{z}$ and $\lambda \in(\bar{\lambda}, 1]$, the ex ante utility of the uninformed agents is $\mathcal{U}_{U}(\lambda)=E_{\bar{\mu}}\left[-e^{-\tau \hat{W}_{U}}\right]$. By equation B.12], we need to show that the mapping, $\mu \longmapsto$ $\left(J_{\mu}^{\text {buy }}+J_{\mu}^{\mathrm{np}}+J_{\mu}^{\text {sell }}\right)$ is decreasing when $\mu_{z}$ is sufficiently large and $\lambda$ is sufficiently close to one. By equation B.12 and the definition of $I_{\mu}^{\ell}$ in equation B.9.

$$
\begin{aligned}
& \frac{\partial}{\partial \mu} J_{\mu}^{\text {buy }} \\
& =-\zeta\left(\underline{\mu} ; \mu_{z}\right) \frac{(\mu-\underline{\mu})(1-\chi)^{2}}{\omega_{f \mid s}} e^{\frac{(\underline{\mu}-\mu)^{2}(1-\chi)^{2}}{2 \omega_{f} \mid s}} \Phi\left(\frac{\kappa}{\sqrt{\omega_{s}}}\left(\frac{\omega_{s}}{\omega_{z}} \mu_{z}-\gamma_{1}(\mu-\underline{\mu})\right)\right) \\
& +\zeta\left(\underline{\mu} ; \mu_{z}\right) e^{\frac{(\underline{\mu}-\mu)^{2}(1-\chi)^{2}}{2 \omega_{f} \mid s}} \phi\left(\frac{\kappa}{\sqrt{\omega_{s}}}\left(\frac{\omega_{s}}{\omega_{z}} \mu_{z}-\gamma_{1}(\mu-\underline{\mu})\right)\right) \frac{\kappa}{\sqrt{\omega_{s}}} \gamma_{1} \\
& +\zeta\left(\underline{\mu} ; \mu_{z}\right) \gamma_{0} \frac{\delta^{2}\left(\frac{\omega_{s}}{\omega_{z}} \mu_{z}+\gamma_{0}(\mu-\underline{\mu})\right)}{\omega_{f \mid s}+\delta^{2} \omega_{s}} e^{\frac{(\underline{\mu}-\mu)^{2}(1-\chi)^{2}}{2 \omega_{f} \mid s}} \Phi\left(\frac{\kappa}{\sqrt{\omega_{s}}}\left(\frac{\omega_{s}}{\omega_{z}} \mu_{z}-\gamma_{1}(\mu-\underline{\mu})\right)\right) \\
& \equiv \zeta\left(\underline{\mu} ; \mu_{z}\right)\left(j_{1}^{\text {buy }}+j_{2}^{\text {buy }}+j_{3}^{\text {buy }}\right),
\end{aligned}
$$

and,

$$
\frac{\partial}{\partial \mu} J_{\mu}^{\mathrm{np}}=\zeta_{\mu_{z}} \frac{\lambda}{\tau \omega_{\epsilon} \sqrt{\omega_{s}}} \zeta_{\mu_{z}}^{-1}\left[\phi\left(\frac{\bar{s}-m_{s}(\mu)}{\sqrt{\omega_{s}}}\right)-\phi\left(\frac{\underline{s}-m_{s}(\mu)}{\sqrt{\omega_{s}}}\right)\right] \equiv \zeta_{\mu_{z}}\left(j_{1}^{\mathrm{np}}+j_{2}^{\mathrm{np}}\right),
$$


and,

$$
\begin{aligned}
& \frac{\partial}{\partial \mu} J_{\mu}^{\text {sell }} \\
& =\zeta\left(\bar{\mu} ; \mu_{z}\right) \frac{(\bar{\mu}-\mu)(1-\chi)^{2}}{\omega_{f \mid s}} e^{\frac{(\bar{\mu}-\mu)^{2}(1-\chi)^{2}}{2 \omega_{f \mid s}}}\left[1-\Phi\left(\frac{\kappa}{\sqrt{\omega_{s}}}\left(\frac{\omega_{s}}{\omega_{z}} \mu_{z}+\gamma_{1}(\bar{\mu}-\mu)\right)\right)\right] \\
& -\zeta\left(\bar{\mu} ; \mu_{z}\right) e^{\frac{(\bar{\mu}-\mu)^{2}\left(1-\chi_{1}\right)^{2}}{2 \omega_{f \mid s}}} \phi\left(\frac{\kappa}{\sqrt{\omega_{s}}}\left(\frac{\omega_{s}}{\omega_{z}} \mu_{z}+\gamma_{1}(\bar{\mu}-\mu)\right)\right) \frac{\kappa}{\sqrt{\omega_{s}}} \gamma_{1} \\
& +\zeta\left(\bar{\mu} ; \mu_{z}\right) \gamma_{0} \frac{\delta^{2}\left(\frac{\omega_{s}}{\omega_{z}} \mu_{z}+\gamma_{0}(\mu-\bar{\mu})\right)}{\omega_{f \mid s}+\delta^{2} \omega_{s}} e^{\frac{(\bar{\mu}-\mu)^{2}(1-\chi)^{2}}{2 \omega_{f} \mid s}}\left[1-\Phi\left(\frac{\kappa}{\sqrt{\omega_{s}}}\left(\frac{\omega_{s}}{\omega_{z}} \mu_{z}+\gamma_{1}(\bar{\mu}-\mu)\right)\right)\right] \\
& \equiv \zeta\left(\bar{\mu} ; \mu_{z}\right)\left(j_{1}^{\text {sell }}+j_{2}^{\text {sell }}+j_{3}^{\text {sell }}\right),
\end{aligned}
$$

where:

$$
\zeta_{\mu_{z}}=\exp \left(-\frac{\delta^{2}\left(\frac{\omega_{s}}{\omega_{z}} \mu_{z}\right)^{2}}{2\left(\omega_{f \mid s}+\delta^{2} \omega_{s}\right)}\right), \quad \zeta\left(x ; \mu_{z}\right) \equiv \kappa \exp \left(-\frac{\delta^{2}\left(\frac{\omega_{s}}{\omega_{z}} \mu_{z}+\gamma_{0}(\mu-x)\right)^{2}}{2\left(\omega_{f \mid s}+\delta^{2} \omega_{s}\right)}\right) .
$$

We have that: for all $\mu>\underline{\mu}, \lim _{\mu_{z} \rightarrow \infty} j_{1}^{\text {buy }}<0, \lim _{\mu_{z} \rightarrow \infty} j_{2}^{\text {buy }}=0$ and $\lim _{\mu_{z} \rightarrow \infty j} j_{1}^{\text {sell }}=\lim _{\mu_{z} \rightarrow \infty} j_{2}^{\text {sell }}=0$. Note, also, that $\lim _{\mu_{z} \rightarrow \infty} \frac{\partial}{\partial \mu} J_{\mu}^{\mathrm{np}} \leq 0$ for all $\mu$, which it does whenever $\lim _{\mu_{z} \rightarrow \infty} j_{1}^{\mathrm{np}}=0$. It is the case, since straightforward calculations leave: $j_{1}^{\text {np }} \propto e^{-\left(c_{1} \mu_{z}^{2}+c_{2} \mu_{z}+c_{3}\right)}$ for three constants $c_{i}$, and $c_{1}$ strictly positive. Therefore, there exists a $\breve{\mu}_{z}>0$ such that, for all $\mu_{z}>\breve{\mu}_{z}$, we have $j_{1}^{\text {buy }}+j_{2}^{\text {buy }}+j_{1}^{\text {sell }}+j_{2}^{\text {sell }}+j_{1}^{\text {np }}+j_{2}^{\text {np }}<0$. Next, we write

$$
\begin{aligned}
& \frac{\partial}{\partial \mu}\left(J_{\mu}^{\text {buy }}+J_{\mu}^{\mathrm{np}}+J_{\mu}^{\text {sell }}\right) \\
& =\zeta_{\mu_{z}} \cdot\left(\frac{\zeta\left(\underline{\mu} ; \mu_{z}\right)}{\zeta_{\mu_{z}}}\left(j_{1}^{\text {buy }}+j_{2}^{\text {buy }}+j_{3}^{\text {buy }}\right)+j_{1}^{\text {np }}+j_{2}^{\text {np }}+\frac{\zeta\left(\bar{\mu} ; \mu_{z}\right)}{\zeta_{\mu_{z}}}\left(j_{1}^{\text {sell }}+j_{2}^{\text {sell }}+j_{3}^{\text {sell }}\right)\right) \\
& \equiv \zeta_{\mu_{z}} \cdot \bar{j} .
\end{aligned}
$$

Because $\lim _{\lambda \rightarrow 1} \gamma_{0}=0$ and, hence, for all finite $\mu_{z}, \lim _{\lambda \rightarrow 1} j_{3}^{\text {buy }}=\lim _{\lambda \rightarrow 1} j_{3}^{\text {sell }}=0$ and $\lim _{\lambda \rightarrow 1} \zeta\left(x ; \mu_{z}\right)=\zeta_{\mu_{z}}$ for all $x$, such that for any finite $\mu_{z}$ larger than $\breve{\mu}_{z}$ there exists $\bar{\lambda}$ depending on $\mu_{z}$ such that $\bar{j}<0$. Therefore, we have shown that for all $\mu$ strictly higher than $\mu$, the function $J_{\mu} \equiv J_{\mu}^{\text {buy }}+J_{\mu}^{\mathrm{np}}+J_{\mu}^{\text {sell }}$ is decreasing in $\mu$. Because $J_{\mu}$ is continuous in the closed and bounded interval $[\bar{\mu}, \bar{\mu}]$, then, by Weierstrass theorem, $J_{\mu}$ takes its absolute maximum on $[\mu, \bar{\mu}]$. Suppose that $J_{\mu}$ is the global maximum, and then the proof follows. Suppose that $J_{\mu}$ is not the global maximum; then, there exists an open interval of $[\mu, \bar{\mu}]$ on which $J_{\mu}$ is increasing in $\mu$, which contradicts that $J_{\mu}$ is decreasing in $\mu$ for all $\mu>\mu$.

Proof of Proposition 3. We show that for all $\mu_{z} \geq \bar{\mu}_{z}$, where $\bar{\mu}_{z}$ is as in Lemma $1, \mathcal{G}(c, 1)>\mathcal{G}(c, 0)$, or equivalently

$$
\frac{\mathcal{U}_{I}(c, 0)}{\mathcal{U}_{U}(0)}>\frac{\mathcal{U}_{I}(c, 1)}{\mathcal{U}_{U}(1)}
$$

We utilize the expressions for $I_{\mu}^{\text {buy }}, I_{\mu}^{\mathrm{np}}$ and $I_{\mu}^{\text {sell }}$ given in Appendix B and calculate that for $\lambda=0$,

$$
\begin{aligned}
& J_{\underline{\mu}}^{\mathrm{buy}}=I_{\underline{\mu}}^{\mathrm{buy}}=-c_{0} \exp \left(-\frac{\tau^{2} \omega_{f} c_{0}^{2}}{2} \mu_{z}^{2}\right) \Phi\left(\frac{c_{0}}{\sqrt{\omega_{z}}} \mu_{z}\right), \\
& J_{\underline{\mu}}^{\mathrm{np}}=I_{\underline{\mu}}^{\mathrm{np}}=0, \\
& J_{\underline{\mu}}^{\mathrm{sell}}=\exp \left(\frac{\Delta \mu^{2}}{2 \omega_{f}}\right) I_{\underline{\mu}}^{\text {sell }}, \quad I_{\underline{\mu}}^{\mathrm{sell}}=-c_{0} \exp \left(-\frac{c_{0}^{2}}{2} \frac{\left(\tau \omega_{f} \mu_{z}-\Delta \mu\right)^{2}}{\omega_{f}}\right) \Phi\left(-\frac{c_{0}}{\sqrt{\omega_{z}}}\left(\mu_{z}+\tau \omega_{z} \Delta \mu\right)\right) .
\end{aligned}
$$

Therefore, for $\mu_{z}$ large enough, and Lemma 1,

$$
\frac{\mathcal{U}_{I}(c, 0)}{\mathcal{U}_{U}(0)}=e^{\tau c} \sqrt{\frac{\omega_{\epsilon}}{\omega_{f}}} \frac{I_{\underline{\mu}}^{\text {buy }}+I_{\underline{\mu}}^{\text {sell }}}{J_{\underline{\mu}}^{\text {buy }}+J_{\underline{\mu}}^{\text {sell }}}=e^{\tau c} \sqrt{\frac{\omega_{\epsilon}}{\omega_{f}}} \frac{1+\frac{I_{\underline{\mu}}^{\text {sell }}}{I_{\underline{\mu}}^{\text {buy }}}}{1+\exp \left(\frac{\Delta \mu^{2}}{2 \omega_{f}}\right) \frac{I_{\underline{\mu}}^{\text {sell }}}{I_{\underline{\mu}}^{\text {buy }}}},
$$


where $\lim _{\mu_{z} \uparrow \infty}\left(I_{\underline{\mu}}^{\text {sell }} / I_{\underline{\mu}}^{\text {buy }}\right)=0$, by the L'Hôpital's rule. Therefore,

$$
\lim _{\mu_{z} \uparrow \infty} \frac{\mathcal{U}_{I}(c, 0)}{\mathcal{U}_{U}(0)}=e^{\tau c} \sqrt{\frac{\omega_{\epsilon}}{\omega_{f}}}
$$

Next, we show that, for $\lambda=1$,

$$
\lim _{\mu_{z} \uparrow \infty} \frac{\mathcal{U}_{I}(c, 1)}{\mathcal{U}_{U}(1)}=e^{\tau c} \sqrt{\frac{\omega_{\epsilon}}{\omega_{f \mid s, \lambda=1}}} \exp \left(-\frac{\Delta \mu^{2}\left(1-\chi_{1}\right)^{2}}{2 \omega_{f \mid s, \lambda=1}}\right),
$$

where $\omega_{f \mid s, \lambda=1}=\lim _{\lambda \rightarrow 1} \omega_{f \mid s}$ and $\chi_{1}=\lim _{\lambda \rightarrow 1} \chi$. By the expressions for $J_{\mu}^{\ell}$ and $I_{\mu}^{\ell}$ in Appendix B and Lemma 2, we have that for $\mu_{z}$ large enough,

$$
\begin{aligned}
J_{\bar{\mu}}^{\text {buy }} & =\exp \left(\frac{\Delta \mu^{2}\left(1-\chi_{1}\right)^{2}}{2 \omega_{f \mid s, \lambda=1}}\right) \cdot \mathcal{I}_{1} \Phi\left(\frac{\kappa}{\sqrt{\omega_{s, \lambda=1}}}\left(\frac{\omega_{s, \lambda=1}}{\omega_{z}} \mu_{z}-\bar{\gamma} \Delta \mu\right)\right), \\
J_{\bar{\mu}}^{\mathrm{np}} & =-\left[\Phi\left(\frac{\bar{s}_{1}-m_{s}(\bar{\mu})}{\sqrt{\omega_{s, \lambda=1}}}\right)-\Phi\left(\frac{\underline{s}_{1}-m_{s}(\bar{\mu})}{\sqrt{\omega_{s, \lambda=1}}}\right)\right], \\
J_{\bar{\mu}}^{\text {sell }} & =\mathcal{I}_{1}\left[1-\Phi\left(\frac{\kappa}{\sqrt{\omega_{s, \lambda=1}}} \frac{\omega_{s, \lambda=1}}{\omega_{z}} \mu_{z}\right)\right],
\end{aligned}
$$

for some $\bar{\gamma}>0$ independent of $\mu_{z}$, where $\omega_{s, \lambda=1}=\lim _{\lambda \rightarrow 1} \omega_{s}, \bar{s}_{1}=\lim _{\lambda \rightarrow 1} \bar{s}, \underline{s}_{1}=\lim _{\lambda \rightarrow 1} \underline{s}$, and $\mathcal{I}_{1}$ is as in equation C.5 in the proof of Lemma 2. Hence, we have

$$
\frac{\mathcal{U}_{I}(c, 1)}{\mathcal{U}_{U}(1)}=e^{\tau c} \sqrt{\frac{\omega_{\epsilon}}{\omega_{f \mid s, \lambda=1}}} \frac{\mathcal{I}_{1}}{J_{\bar{\mu}}^{\text {buy }}+J_{\bar{\mu}}^{\mathrm{np}}+J_{\bar{\mu}}^{\text {sell }}}=e^{\tau c} \sqrt{\frac{\omega_{\epsilon}}{\omega_{f \mid s, \lambda=1}}} \exp \left(-\frac{\Delta \mu^{2}\left(1-\chi_{1}\right)^{2}}{2 \omega_{f \mid s, \lambda=1}}\right) \frac{1}{\mathcal{Z}\left(\mu_{z}\right)},
$$

where, by straightforward calculations,

$$
\begin{aligned}
\mathcal{Z}\left(\mu_{z}\right) & \equiv \Phi\left(\frac{\kappa}{\sqrt{\omega_{s, \lambda=1}}}\left(\frac{\omega_{s, \lambda=1}}{\omega_{z}} \mu_{z}-\bar{\gamma} \Delta \mu\right)\right) \\
& +\left[\frac{J_{\bar{\mu}}^{\mathrm{np}}}{\mathcal{I}_{1}}+\Phi\left(-\frac{\kappa}{\sqrt{\omega_{s, \lambda=1}}} \frac{\omega_{s, \lambda=1}}{\omega_{z}} \mu_{z}\right)\right] \exp \left(-\frac{\Delta \mu^{2}\left(1-\chi_{1}\right)^{2}}{2 \omega_{f \mid s, \lambda=1}}\right) .
\end{aligned}
$$

We have, by the L'Hôpital's rule,

$$
\lim _{\mu_{z} \uparrow \infty} \frac{J_{\bar{\mu}}^{\mathrm{np}}}{\mathcal{I}_{1}}=\bar{c} \cdot \lim _{\mu_{z} \uparrow \infty} \frac{\phi\left(\frac{\bar{s}_{1}-m_{s}(\bar{\mu})}{\sqrt{\omega_{s, \lambda=1}}}\right)-\phi\left(\frac{\underline{s}_{1}-m_{s}(\bar{\mu})}{\sqrt{\omega_{s, \lambda=1}}}\right)}{\mu_{z} e^{-\frac{1}{2} \tau^{2} \omega_{\epsilon} c_{1}^{2} \mu_{z}^{2}}}=0,
$$

for some constant $\bar{c}>0$, where the last equality follows by comparing the loadings of $\mu_{z}^{2}$ of the exponentials in the numerator and the denominator, and using the definition of $c_{1}$ in equation $C .5$. Therefore, $\lim _{\mu_{z} \uparrow \infty} \mathcal{Z}\left(\mu_{z}\right)=1$ such that equation C.8 holds, due to equation C.9. Proposition III follows from equation C.7 and equation C.8 and $\Delta \mu$ large enough.

Proof of equation 5.7. We rely on the following lemma, which we give without proof, as it can be derived by well known properties of the normal distribution.

Lemma C.1. Let $\mathcal{F}_{s}$ and $\mathcal{F}_{t}$ be two nested information sets, $\mathcal{F}_{s} \subseteq \mathcal{F}_{t}$, and let $R$ be normally distributed with respect to $\mathcal{F}_{s}$ and $\mathcal{F}_{t}$, and assume $\operatorname{Var}\left(R \mid \mathcal{F}_{t}\right)$ is constant. Then,

$$
x\left(\mathcal{F}_{t}\right) \equiv \arg \max _{x} E\left(-e^{-\tau x R} \mid \mathcal{F}_{t}\right)=\arg \max _{x} x\left(E\left(R \mid \mathcal{F}_{t}\right)-x \frac{\tau}{2} \operatorname{Var}\left(R \mid \mathcal{F}_{t}\right)\right)=\frac{E\left(R \mid \mathcal{F}_{t}\right)}{\tau \operatorname{Var}\left(R \mid \mathcal{F}_{t}\right)},
$$

and

$$
E\left[-\exp \left(-\tau x\left(\mathcal{F}_{t}\right) R\right) \mid \mathcal{F}_{s}\right]=-\left(\frac{\operatorname{Var}\left(R \mid \mathcal{F}_{t}\right)}{\operatorname{Var}\left(R \mid \mathcal{F}_{s}\right)}\right)^{1 / 2} \exp \left(-\frac{E\left(R \mid \mathcal{F}_{s}\right)^{2}}{2 \operatorname{Var}\left(R \mid \mathcal{F}_{s}\right)}\right)
$$

As for the proof of equation 5.7, we have that in the no-ambiguity benchmark, $\Delta \mu=0$, asset returns are unconditionally normally distributed, so Lemma C.1 implies that the ex ante utility deriving from the unconditional portfolio choice $x_{0}$ is,

$$
\mathcal{U}_{0}(\lambda)=-\exp \left(-\frac{1}{2} \frac{E(R)^{2}}{\operatorname{Var}(R)}\right)
$$




\section{MELE \& SANGIORGI UNCERTAINTYAND INFORMATION IN ASSET MARKETS 1563}

Next, let $\mathcal{F}_{i}$ denote agent- $i$ information set at the trading stage and, accordingly, let $x_{i}\left(\mathcal{F}_{i}\right)$ be his optimal demand conditional on $\mathcal{F}_{i}$ and $\mathcal{U}_{i}(\lambda)$ his ex ante utility. If the asset return $R$ is normally distributed with respect to $\mathcal{F}_{i}$ and also unconditionally, then,

$$
\mathcal{U}_{i}(\lambda)=-\left(\frac{\operatorname{Var}\left(R \mid \mathcal{F}_{i}\right)}{\operatorname{Var}(R)}\right)^{1 / 2} \exp \left(-\frac{1}{2} \frac{E(R)^{2}}{\operatorname{Var}(R)}\right)=\left(\frac{\operatorname{Var}\left(R \mid \mathcal{F}_{i}\right)}{\operatorname{Var}(R)}\right)^{1 / 2} \mathcal{U}_{0}(\lambda)
$$

where the first equality holds by Lemma C.1 and second from the expression of $\mathcal{U}_{0}(\lambda)$ in equation C.11. Given returns $R$ are normally distributed both conditional on price information and unconditionally for $\Delta \mu=0$, these results imply that equation 5.7 holds.

Finally, we derive the unconditional portfolio choice in equation 5.5 in the general case of a market with ambiguity. We have:

Proposition C.1. The unconditional portfolio choice, $x_{0}$, is implicitly defined by

$$
x_{0}= \begin{cases}\frac{E_{\underline{\mu}}\left(\frac{E_{\underline{\mu}}(R \mid s)}{\tau \omega_{f \mid s}} e^{-\tau x_{0} E_{\underline{\mu}}(R \mid s)}\right)}{E_{\underline{\mu}}\left(e^{-\tau x_{0} E_{\underline{\mu}}(R \mid s)}\right)}, & \text { for } E_{\underline{\mu}}(R)>0 \\ 0, & \text { for } E_{\underline{\mu}}(R) \leq 0 \text { and } E_{\bar{\mu}}(R) \geq 0 \\ \frac{E_{\bar{\mu}}\left(\frac{E_{\bar{\mu}}(R \mid s)}{\tau \omega} e^{-\tau \mid s} e^{-\tau E_{\bar{\mu}}(R \mid s)}\right)}{E_{\bar{\mu}}\left(e^{-\tau x_{0} E_{\bar{\mu}}(R \mid s)}\right)}, & \text { for } E_{\bar{\mu}}(R)<0\end{cases}
$$

Moreover, $x_{0} \in\left(0, \frac{E_{\mu}(R)}{\tau \omega_{f \mid s}}\right)$ for $E_{\underline{\mu}}(R)>0$ and $x_{0} \in\left(\frac{E_{\bar{\mu}}(R)}{\tau \omega_{f \mid s}}, 0\right)$ for $E_{\bar{\mu}}(R)<0$.

We prove Proposition C.1 through the following lemma.

Lemma C.2. The inner minimization problem in equation 5.5 is solved by $\mu=\underline{\mu}$ for $x_{0}>0$ and by $\mu=\bar{\mu}$ for $x_{0}<0$.

Proof. Note that,

$$
\begin{aligned}
& \min _{\mu \in[\underline{\mu}, \bar{\mu}]} E_{\mu}\left[-e^{-\tau x R}\right] \\
= & \min _{\mu \in[\underline{\mu}, \bar{\mu}]} E_{\mu}\left[E_{\mu}\left[e^{-\tau x\left(E(R \mid s)-x \frac{\tau}{2} \operatorname{Var}(R \mid s)\right)} \mid s\right]\right] \\
= & \min _{\mu \in[\underline{\mu}, \bar{\mu}]} E_{\mu}\left[-e^{-\tau x\left(\left(\mu_{\theta \mid s}(s ; \mu)-P(s)\right)-x \frac{\tau}{2} \omega_{f \mid s}\right)}\right] \\
= & \min _{\mu \in[\underline{\mu}, \bar{\mu}]}\left[\int_{-\infty}^{\underline{s}} h(t, \mu, x) d t+\int_{\underline{s}}^{\bar{s}} h(t, \mu, x) d t+\int_{\bar{s}}^{\infty} h(t, \mu, x) d t\right],
\end{aligned}
$$

where

$$
h(t, \mu, x) \equiv-e^{-\tau x\left(\left(\mu_{\theta \mid s}(t ; \mu)-P(t)\right)-x \frac{\tau}{2} \omega_{f \mid s}\right)} f\left(t ; m_{s}(\mu), \omega_{s}\right),
$$

and $m_{s}(\mu)$ is defined in B.4. The integrand $h(t, \mu, x)$ is a continuous function of $t$ and $\mu$ over the ranges of integration, and so is its partial derivative with respect to $\mu$,

$$
\begin{aligned}
& \frac{\partial}{\partial \mu} h(t, \mu, x) \\
& =e^{-\tau x\left(\left(\mu_{\theta \mid s}(t ; \mu)-P(t)\right)-x \frac{\tau}{2} \omega_{f \mid s}\right)}\left(\tau x(1-\chi) f\left(t ; m_{s}(\mu), \omega_{s}\right)+\frac{\lambda}{\tau \omega_{\epsilon}} \frac{\partial}{\partial t} f\left(t ; m_{s}(\mu), \omega_{s}\right)\right) .
\end{aligned}
$$

Hence, differentiating under the integral sign and rearranging, leaves:

$$
\begin{aligned}
& \frac{\partial}{\partial \mu} E_{\mu}\left(-e^{-\tau x R}\right) \\
& =\int_{-\infty}^{\infty} e^{-\tau x\left(\left(\mu_{\theta \mid s}(t ; \mu)-P(t)\right)-x \frac{\tau}{2} \omega_{f \mid s}\right)}\left(\tau x(1-\chi) f\left(t ; m_{s}(\mu), \omega_{s}\right)+\frac{\lambda}{\tau \omega_{\epsilon}} \frac{\partial}{\partial t} f\left(t ; m_{s}(\mu), \omega_{s}\right)\right) d t .
\end{aligned}
$$


Integrating the second term by parts and simplifying gives,

$$
\begin{aligned}
& \int_{-\infty}^{\infty} e^{-\tau x\left(\left(\mu_{\theta \mid s}(t ; \mu)-P(t)\right)-x \frac{\tau}{2} \omega_{f \mid s}\right)} \frac{\lambda}{\tau \omega_{\epsilon}} \frac{\partial}{\partial t} f\left(t ; m_{s}(\mu), \omega_{s}\right) d t \\
& =-\int_{-\infty}^{\infty}\left(\frac{\partial}{\partial t} e^{-\tau x\left(\left(\mu_{\theta \mid s}(t ; \mu)-P(t)\right)-x \frac{\tau}{2} \omega_{f \mid s}\right)}\right) \frac{\lambda}{\tau \omega_{\epsilon}} f\left(t ; m_{s}(\mu), \omega_{s}\right) d t \\
& =\int_{-\infty}^{\infty} \tau x\left(\chi-\frac{\lambda}{\tau \omega_{\epsilon}} \frac{\partial}{\partial t} P(t)\right) e^{-\tau x\left(\left(\mu_{\theta \mid s}(t ; \mu)-P(t)\right)-x \frac{\tau}{2} \omega_{f \mid s}\right)} f\left(t ; m_{s}(\mu), \omega_{s}\right) d t
\end{aligned}
$$

Plugging back into equation C.13 yields:

$$
\begin{aligned}
& \frac{\partial}{\partial \mu} E_{\mu}\left(-e^{-\tau x R}\right) \\
& =\tau x \int_{-\infty}^{\infty} e^{-\tau x\left(\left(\mu_{\theta \mid s}(t ; \mu)-P(t)\right)-x \frac{\tau}{2} \omega_{f \mid s}\right)}\left(1-\frac{\lambda}{\tau \omega_{\epsilon}} \frac{\partial}{\partial t} P(t)\right) f\left(t ; m_{s}(\mu), \omega_{s}\right) d t
\end{aligned}
$$

By the equilibrium price function defined by equation 3.7, it is immediate that $\frac{\lambda}{\tau \omega_{\epsilon}} \frac{\partial}{\partial t} P(t) \leq 1$, with the inequality being strict outside the non-participation region. Therefore, we have:

$$
\operatorname{sign}\left(\frac{\partial}{\partial \mu} E_{\mu}\left(-e^{-\tau x R}\right)\right)=\operatorname{sign}(x)
$$

which proves the original claim.

Proof of Proposition C.1. We begin by proving that the maximization problem in equation 5.5 has a strictly positive solution $x_{0}>0$ if and only if $E_{\mu}(R)>0$. By Lemma C. 2 we look for $x_{0}>0$ which solves

$$
x_{0}=\arg \max _{x} E_{\underline{\mu}}\left(-e^{-\tau x R}\right)=\arg \max _{x} E_{\underline{\mu}}\left(e^{-\tau x\left(E_{\underline{\mu}}(R \mid s)-x \frac{\tau}{2} \omega_{f \mid s}\right)}\right) .
$$

As a first step we show that we can differentiate under the integral sign the maximand in equation C.14,

$$
\begin{aligned}
E_{\underline{\mu}}\left(e^{-\tau x\left(E_{\underline{\mu}}(R \mid s)-x \frac{\tau}{2} \omega_{f \mid s}\right)}\right) & =\int_{-\infty}^{\infty} e^{-\tau x\left(\left(\mu_{\theta \mid s}(t ; \underline{\mu})-P(t)\right)-x \frac{\tau}{2} \omega_{f \mid s}\right)} f\left(t ; m_{s}(\underline{\mu}), \omega_{s}\right) d t \\
& =\int_{-\infty}^{\underline{s}} h(t, \underline{\mu}, x) d t+\int_{\underline{s}}^{\bar{s}} h(t, \underline{\mu}, x) d t+\int_{\bar{s}}^{\infty} h(t, \underline{\mu}, x) d t
\end{aligned}
$$

which we can, as $h(t, \underline{\mu}, x)$ and $\frac{\partial}{\partial x} h(t, \underline{\mu}, x)$ are continuous functions of $t$ and $x$ over the ranges of integration. Because $E_{\mu}(R \mid s)=\mu_{\theta \mid s}(s ; \underline{\mu})-P(s)$ is linear in $s$ over the very same ranges of integration, the expression $E_{\underline{\mu}}\left(E_{\underline{\mu}}(R \mid s) e^{-\tau x\left(E_{\underline{\mu}}(R \mid s)-x \frac{\tau}{2} \omega_{f \mid s}\right)}\right)$ is also differentiable under the integral sign. Then, the first-order condition for the maximization problem in equation 5.5 for $x>0$ gives

$$
G(x) \equiv E_{\underline{\mu}}\left[\left(E_{\underline{\mu}}(R \mid s)-x \tau \omega_{f \mid s}\right) e^{-\tau x\left(E_{\underline{\mu}}(R \mid s)-x \frac{\tau}{2} \omega_{f \mid s}\right)}\right]=0,
$$

with second-order condition

$$
G^{\prime}(x)=-\tau E_{\underline{\mu}}\left[\left(\omega_{f \mid s}+\left(E_{\underline{\mu}}(R \mid s)-x \tau \omega_{f \mid s}\right)^{2}\right) e^{-\tau x\left(E_{\underline{\mu}}(R \mid s)-x \frac{\tau}{2} \omega_{f \mid s}\right)}\right]<0 .
$$

Notice that

$$
G(0)=E_{\underline{\mu}}\left(E_{\underline{\mu}}(R \mid s)\right)=E_{\underline{\mu}}(R)
$$

which, together with equation $C .16$ implies that a strictly positive solution to $G(x)=0$ exists only if $E_{\underline{\mu}}(R)>0$. Hence, take $E_{\mu}(R)>0$ and define

$$
\bar{x} \equiv \frac{E_{\underline{\mu}}(R)}{\tau \omega_{f \mid s}} .
$$

We will show that $G(\bar{x})<0$, or equivalently,

$$
G(\bar{x})<0 \Leftrightarrow E_{\underline{\mu}}\left[\left(E_{\underline{\mu}}(R \mid s)-\bar{x} \tau \omega_{f \mid s}\right) e^{-\tau \bar{x} E_{\underline{\mu}}(R \mid s)}\right]<0 \Leftrightarrow E_{\underline{\mu}}(R)>E_{\underline{\mu}}^{\tilde{P}}\left(E_{\underline{\mu}}(R \mid s)\right),
$$

where

$$
\left.\frac{d \tilde{P}}{d P}\right|_{\mathcal{F}(s)} \equiv \frac{e^{-\tau \bar{x} E_{\underline{\mu}}(R \mid s)}}{E\left[e^{-\tau \bar{x} E_{\underline{\mu}}(R \mid s)}\right]}
$$

Given that $E_{\mu}(R \mid s)$ is a strictly decreasing function of $s$ and $\bar{x}>0$, the measure $\tilde{P}$ assigns larger weight to states $s$ in which $E_{\underline{\mu}}(R \mid s)$ is negative and lower weight to states $s$ in which $E_{\underline{\mu}}(R \mid s)$ is positive, which implies 
MELE \& SANGIORGI UNCERTAINTYAND INFORMATION IN ASSET MARKETS 1565

$E_{\underline{\mu}}^{\tilde{P}}\left(E_{\underline{\mu}}(R \mid s)\right)<E_{\underline{\mu}}\left(E_{\underline{\mu}}(R \mid s)\right)$ and therefore the last inequality in C.17. Since $G(0)>0$ and $G(\bar{x})<0$, then there exists a value $x_{0} \in\left(0, \frac{E_{\mu}(R)}{\tau \omega_{f \mid s}}\right)$ that solves equation $\mathbf{C . 1 5}$; since $G^{\prime}(x)<0$ the solution to equation $\mathbf{C} .15$ is unique. Rearranging equation C.15 and simplifying gives

$$
x_{0}=\frac{E_{\underline{\mu}}\left(\frac{E_{\underline{\mu}}(R \mid s)}{\tau \omega_{f \mid s}} e^{-\tau x_{0} E_{\underline{\mu}}(R \mid s)}\right)}{E_{\underline{\mu}}\left(e^{-\tau x_{0} E_{\underline{\mu}}(R \mid s)}\right)} .
$$

The case for $E_{\bar{\mu}}(R)<0$ and $x_{0}<0$ is similar ad is omitted. Finally, for both $E_{\underline{\mu}}(R) \leq 0$ and $E_{\bar{\mu}}(R) \geq 0$, the function $\min _{\mu \in[\mu, \bar{\mu}]} E_{\mu}\left(-e^{-\tau x R}\right)$ is decreasing (increasing) in $x$ for all $x \geq 0(x \leq 0)$ such that the unconditional portfolio problem is solved by $x_{0}=0$.

Finally, to prove Proposition 4 we rely on the following lemma:

Lemma C.3. Let $\lambda>0$. Then for each $\ell \in \mathbb{R}$, there exists a $\hat{\mu}_{z}>0$ depending on $\ell$ such that for all $\mu_{z} \geq \hat{\mu}_{z}$, the equilibrium price in equation 3.7 satisfies $E_{\mu}(P(s))<\ell$ for all $\mu \in[\underline{\mu}, \bar{\mu}]$.

Proof. By market clearing, and the definition of the compound signal $s$ in equation 3.5, the equilibrium price satisfies:

$$
P(s)=\left(\frac{\lambda}{\tau \omega_{\epsilon}}\right)^{-1}\left(-\mu_{z}+s+(1-\lambda) \frac{\mu_{f \mid s}\left(s ; \mu^{*}(s)\right)-P(s)}{\tau \omega_{f \mid s}}\right),
$$

where, using equations A.2 A.3 and the expression for the equilibrium price in equation 3.7,

$$
\mu_{f \mid s}\left(s ; \mu^{*}(s)\right)-P(s)=\left\{\begin{array}{ll}
\delta(\underline{s}-s), & \text { for } s<\underline{s} \\
0, & \text { for } s \in[\underline{s}, \bar{s}] \\
\delta(\bar{s}-s), & \text { for } s>\bar{s}
\end{array} \quad\right.
$$

where $\delta$ is as in the first of equation B.8. Plugging equation C.19 into equation C.18, and taking expectation under a generic prior $\mu$,

$$
\begin{aligned}
& \frac{\lambda}{\tau \omega_{\epsilon}} \cdot E_{\mu}(P(s)) \\
& =-\mu_{z}+m_{s}(\mu)+(1-\lambda) \delta \frac{E_{\mu}\left((\underline{s}-s) \mathbb{I}_{s<\underline{s}}+(\bar{s}-s) \mathbb{I}_{s>\bar{s}}\right)}{\tau \omega_{f \mid s}} \\
& =-\mu_{z}+m_{s}(\mu) \\
& +(1-\lambda) \delta \frac{\underline{s} \Phi\left(\frac{s-m_{s}(\mu)}{\sqrt{\omega_{s}}}\right)+\bar{s}\left(1-\Phi\left(\frac{\bar{s}-m_{s}(\mu)}{\sqrt{\omega_{s}}}\right)\right)-m_{s}(\mu)+\int_{\underline{s}}^{\bar{s}} t d \Phi\left(t ; m_{s}(\mu), \omega_{s}\right)}{\tau \omega_{f \mid s}}
\end{aligned}
$$

where, using the property of the truncated normal distribution,

$$
\begin{aligned}
& \int_{\underline{s}}^{\bar{s}} t d \Phi\left(t ; m_{s}(\mu), \omega_{s}\right) \\
& =m_{s}(\mu)\left(\Phi\left(\frac{\bar{s}-m_{s}(\mu)}{\sqrt{\omega_{s}}}\right)-\Phi\left(\frac{\underline{s}-m_{s}(\mu)}{\sqrt{\omega_{s}}}\right)\right)-\left(\phi\left(\frac{\bar{s}-m_{s}(\mu)}{\sqrt{\omega_{s}}}\right)-\phi\left(\frac{s-m_{s}(\mu)}{\sqrt{\omega_{s}}}\right)\right) \sqrt{\omega_{s}}
\end{aligned}
$$

and $m_{s}(\mu)$ is as in equation B.4. Replacing this expression into equation C.20, and rearranging terms, yields,

$$
\begin{aligned}
& E_{\mu}(P(s)) \\
& =\left(\frac{\lambda}{\tau \omega_{\epsilon}}\right)^{-1}\left[\left(-\mu_{z}+m_{s}(\mu)+\frac{(1-\lambda) \delta \sqrt{\omega_{s}}}{\tau \omega_{f \mid s}}\left(g\left(\frac{\underline{s}-m_{s}(\mu)}{\sqrt{\omega_{s}}}\right)-g\left(\frac{\bar{s}-m_{s}(\mu)}{\sqrt{\omega_{s}}}\right)\right)\right)\right],
\end{aligned}
$$

where for any $t \in \mathbb{R}$, we define the function, $g(t) \equiv t \Phi(t)+\phi(t)$. The claim follows by taking the limits in equation C.21.

Proof of Proposition 4. We first establish that for $\mu_{z}$ large, there exists a $\bar{\lambda} \in(0,1)$, such that the value of price information in equation 5.6,

$$
\mathcal{G}_{p}(\lambda)=-\frac{1}{\tau} \ln \left(\frac{\mathcal{U}_{U}(\lambda)}{\mathcal{U}_{0}(\lambda)}\right)
$$

is continuous in $\lambda$ for all $\lambda \in(\bar{\lambda}, 1]$. By equation B.12 $\mathcal{U}_{U}(\lambda)$ is continuous in $\lambda$ over some some range $(\bar{\lambda}, 1]$ if $\mu_{U}$ is constant over $(\bar{\lambda}, 1]$; indeed, for $\mu_{z}$ large, by Lemma 2 there exists a $\bar{\lambda}$ such that $\mu_{U}=\bar{\mu}$ for all $\lambda \in(\bar{\lambda}, 1]$. By Lemma 
C.3, $E_{\mu}(R)>0$ for $\mu_{z}$ large, and therefore by Proposition C.1 and Lemma C.2, $\mathcal{U}_{0}(\lambda)=E_{\mu}\left(-e^{-\tau x_{0} R}\right)$ for all $\lambda$, where by equation C.15 and the implicit function theorem, $x_{0}$ is a continuous function of $\lambda$ for all $\lambda>0$.

Secondly, we establish that for $\mu_{z}$ large $\mathcal{G}_{p}(1)$ is negative for $\Delta \mu$ sufficiently high. Note that,

$$
\mathcal{G}_{p}(1)=-\frac{1}{\tau} \ln \left(\frac{\mathcal{U}_{U}(1)}{\mathcal{U}_{I}(c, 1)}\right)-\frac{1}{\tau} \ln \left(\frac{\mathcal{U}_{I}(c, 1)}{\mathcal{U}_{0}(1)}\right) .
$$

Moreover, in $\lambda=1$, the asset realized returns are independent of $\theta$, such that by Lemma C.1 and equation C.12,

$$
\frac{\mathcal{U}_{I}(c, 1)}{\mathcal{U}_{0}(1)}=e^{\tau c} \sqrt{\left.\frac{\operatorname{Var}(R \mid \theta, p)}{\operatorname{Var}(R)}\right|_{\lambda=1}}=e^{\tau c} \sqrt{\frac{\omega_{\varepsilon}}{\left.\operatorname{Var}(R)\right|_{\lambda=1}}},
$$

and by equation C.8.

$$
\lim _{\mu_{z} \uparrow \infty} \frac{\mathcal{U}_{U}(1)}{\mathcal{U}_{I}(c, 1)}=e^{-\tau c} \sqrt{\frac{\left.\operatorname{Var}(R \mid p)\right|_{\lambda=1}}{\omega_{\epsilon}}} \exp \left(\frac{\Delta \mu^{2}\left(1-\chi_{1}\right)^{2}}{2 \omega_{f \mid s, \lambda=1}}\right) .
$$

Therefore, taking the limit in equation C.22 and rearranging terms leaves:

$$
\lim _{\mu_{z} \uparrow \infty} \mathcal{G}_{p}(1)=\frac{1}{2 \tau} \ln \left(\frac{\left.\operatorname{Var}(R)\right|_{\lambda=1}}{\left.\operatorname{Var}(R \mid p)\right|_{\lambda=1}}\right)-\frac{\Delta \mu^{2}\left(1-\chi_{1}\right)^{2}}{2 \tau \omega_{f \mid s, \lambda=1}} .
$$

Equation $C .23$ and continuity of $\mathcal{G}_{p}(\lambda)$ on $\lambda \in(\bar{\lambda}, 1]$ imply that there exist values $\overline{\mu_{z}}>0$ and $\overline{\Delta \mu}>0$ such that $\mathcal{G}_{p}(\lambda)<0$ for all $\mu_{z}>\overline{\mu_{z}}$ and $\Delta \mu>\overline{\Delta \mu}$ and $\lambda \in(\bar{\lambda}, 1]$.

Acknowledgments. We wish to thank the Editor, Marco Ottaviani, and four anonymous referees for their invaluable comments and suggestions. We are also deeply indebted to Jacob Sagi for comments, and thank Amil Dasgupta, Diego García, Simon Gervais, Paolo Ghirardato, Christian Julliard, Emre Ozdenoren, Joël Peress, Fabio Trojani, Laura Veldkamp, Michela Verardo and Kathy Yuan for discussions, and seminar participants at Amsterdam Business School, Berkeley-Haas, Bocconi University, Cass Business School, City University of Hong Kong, Imperial College Business School, London School of Economics, Magyar Nemzeti Bank, Universities of Frankfurt, Geneva, Hong Kong, Luxembourg, Roma III and Zurich, Warwick Business School, brown bags at our institutions, the 2010 Financial Intermediation Research Society, the 2010 Western Finance Association, and the 2011 UCLA-SFI conference in Lugano, for additional suggestions. The usual disclaimer applies.

\section{Supplementary Data}

Supplementary data are available at Review of Economic Studies online.

\section{REFERENCES}

AVDIS, E. (2011), "Information Trade-offs in Dynamic Financial Markets" (Working paper Wharton School, University of Pennsylvania).

BARLEVY, G. and VERONESI, P. (2008), “Information Acquisition in Financial Markets: a Correction” (Working paper Chicago).

BREON-DRISH, B. (2010), “Asymmetric Information in Financial Markets: Anything Goes” (Working paper Berkeley, University of Berkeley).

CAO, H., WANG, T. and ZHANG, H. (2005), "Model Uncertainty, Limited Market Participation and Asset Prices”, Review of Financial Studies, 18, 1219-1251.

CARRILlO, J. D. and MARIOTTI, T. (2000), "Strategic Ignorance as a Self-Disciplining Device", Review of Economic Studies, 67, 529-544.

CHAMLEY, C. H. (2007), "Complementarities in Information Acquisition with Short-Term Trades", Theoretical Economics, 2, 441-467.

CHAMLEY, C. H. (2010), "Strategic Complementarity of Information in Financial Markets with Large Shocks", Annals of Finance, 6, 137-145.

CASKEY, J. (2009), "Information in Equity Markets with Ambiguity-Averse Investors", Review of Financial Studies, 22, 3595-3627.

CONDIE, S. and GANGULI, J. (2011), “Ambiguity and Rational Expectations Equilibria”, Review of Economic Studies, 78, 821-845.

CONDIE, S. and GANGULI, J. (2012), “The Pricing Effects of Ambiguous Private Information” (Working paper Brigham Young University).

DOW, J. and WERLANG, S. (1992), "Uncertainty Aversion, Risk Aversion, and the Optimal Choice of Portfolio", Econometrica, 60, 197-204.

EASLEY, D. and O'HARA, M. (2009), “Ambiguity and Non-Participation: the Role of Regulation”, Review of Financial Studies, 22, 1817-1843. 


\section{MELE \& SANGIORGI UNCERTAINTYAND INFORMATION INASSET MARKETS 1567}

EASLEY, D., O’HARA, M. and YANG, L. (2011), “Opaque Trading, Disclosure and Asset Prices: Implications for Hedge Fund Regulation" (Working paper Cornell, Cornell University).

EICHBERGER, J., GRANT, S. and KELSEY, D. (2007), "Updating Choquet Beliefs", Journal of Mathematical Economics, 43, 888-899.

EPSTEIN, L. G. and SCHNEIDER, M. (2003), "Recursive Multiple-Priors", Journal of Economic Theory, 113, 1-31. EPSTEIN, L. G. and SCHNEIDER, M. (2007), "Learning Under Ambiguity”, Review of Economic Studies, 74, $1275-1303$. EPSTEIN, L. G. and SCHNEIDER, M. (2008), "Ambiguity, Information Quality and Asset Pricing", Journal of Finance, 63, 197-228.

GANGULI, J. V. and YANG, L. (2009), "Complementarities, Multiplicity, and Supply Information”, Journal of the European Economic Association, 7, 90-115.

GARCÍA, D. and STROBL, G. (2011), "Relative Wealth Concerns and Complementarities in Information Acquisition", Review of Financial Studies, 24, 169-207.

GHIRARDATO, P., MACCHERONI, F. and MARINACCI, M. (2004), "Differentiating Ambiguity and Ambiguity Attitude", Journal of Economic Theory, 118, 133-173.

GILBOA, I. and MARINACCI, M. (2013), “Ambiguity and the Bayesian Paradigm”, in Acemoglu, D., Arellano, M. and Dekel, E., (eds), Advances in Economics and Econometrics: Theory and Applications, Vol. 1 (Tenth World Congress of the Econometric Society, Cambridge: Cambridge University Press) 179-242.

GILBOA, I. and SCHMEIDLER, D. (1989), "Maxmin Expected Utility with a Non-Unique Prior", Journal of Mathematical Economics, 18, 141-153.

GILBOA, I. and SCHMEIDLER, D. (1993), "Updating Ambiguous Beliefs", Journal of Economic Theory, 59, $33-49$.

GOLLIER, CH. (2011), "Portfolio Choices and Asset Prices: The Comparative Statics of Ambiguity Aversion", Review of Economic Studies, 78, 1329-1344.

GROSSMAN, S. J. and STIGLITZ, J. (1980), "On the Impossibility of Informationally Efficient Markets", American Economic Review, 70, 393-408.

HANANY, E. and KLIBANOFF, P. (2007), "Updating Preferences with Multiple Priors", Theoretical Economics, 2 , 261-298.

HANANY, E. and KLIBANOFF, P. (2009), "Updating Ambiguity Averse Preferences”, The B.E. Journal of Theoretical Economics, 9, (Advances): Article 37.

HELLWIG, CH. and VELDKAMP, L. (2009), "Knowing What Others Know: Coordination Motives in Information Acquisition", Review of Economic Studies, 76, 223-251.

JAFFRAY, J.-Y. (1992), "Bayesian Updating and Belief Functions", IEEE Transactions on Systems, Man and Cybernetics, 22, 1144-1152.

KEYNES, J. M. (1921), A Treatise on Probability. (London: MacMillan and Co).

KLIBANOFF, P., MARINACCI, M., and MUKERJI, S. (2005), "A Smooth Model of Decision Making under Ambiguity", Econometrica, 73, 1849-1492.

KNIGHT, F. H. (1921), Risk, Uncertainty, and Profit. (New York: Houghton Mifflin).

MACHINA, M. J. (1989), "Dynamic Consistency and Non-Expected Utility Models of Choice under Uncertainty", Journal of Economic Literature, 27, 1622-1668.

MANZANO, C. and VIVES, X. (2011), "Public and Private Learning from Prices, Strategic Substitutability and Complementarity, and Equilibrium Multiplicity", Journal of Mathematical Economics, 47, 346-369.

MYATT, D. P. and WALLACE. C. (2012), "Endogenous Information Acquisition in Coordination Games", Review of Economic Studies, 79, 340-374.

OZSOYLEV, H. N. and WERNER, J. (2011), "Liquidity and Asset Prices in Rational Expectations Equilibrium with Ambiguous Information", Economic Theory, 48, 469-491.

PIRES, C. P. (2002), "A Rule for Updating Ambiguous Beliefs", Theory and Decision, 33, 137-152.

RAHI, R. and ZIGRAND, J.-P. (2014), "Information Aggregation in a Competitive Economy" (Working paper London School of Economics).

SCHMEIDLER, D. (1989), "Subjective Probability and Expected Utility without Additivity", Econometrica, 57, $571-587$.

SINISCALCHI, M. (2011), "Dynamic Choice Under Ambiguity", Theoretical Economics, 6, 379-421.

STROTZ, R. H. (1955-1956), "Myopia and Inconsistency in Dynamic Utility Maximization", Review of Economic Studies, 23, 165-180.

VELDKAMP, L. (2006), "Media Frenzies in Markets for Financial Information”, American Economic Review, 96, 577-601. 